\title{
Gain of glycosylation in integrin $\alpha 3$ causes lung disease and nephrotic syndrome
}

\author{
Nayia Nicolaou, ${ }^{1}$ Coert Margadant, ${ }^{2}$ Sietske H. Kevelam, ${ }^{1}$ Marc R. Lilien, ${ }^{3}$ \\ Michiel J.S. Oosterveld, ${ }^{3}$ Maaike Kreft, ${ }^{2}$ Albertien M. van Eerde, ${ }^{1}$ Rolph Pfundt, ${ }^{4}$ Paulien A. Terhal, ${ }^{1}$ \\ Bert van der Zwaag, ${ }^{1}$ Peter G.J. Nikkels, ${ }^{5}$ Norman Sachs, ${ }^{2}$ Roel Goldschmeding, ${ }^{5}$ \\ Nine V.A.M. Knoers, ${ }^{1}$ Kirsten Y. Renkema, ${ }^{1}$ and Arnoud Sonnenberg ${ }^{2}$
}

${ }^{1}$ Department of Medical Genetics, University Medical Center Utrecht, Utrecht, The Netherlands. 2Division of Cell Biology, The Netherlands Cancer Institute, Amsterdam, The Netherlands. ${ }^{3}$ Department of Pediatric Nephrology, University Medical Center Utrecht, Wilhelmina Children's Hospital, Utrecht, The Netherlands. ${ }^{4}$ Department of Human Genetics, Radboud University Nijmegen Medical Center, Nijmegen, The Netherlands. 5Department of Pathology, University Medical Center Utrecht, Utrecht, The Netherlands.

\begin{abstract}
Integrins are transmembrane $\alpha \beta$ glycoproteins that connect the extracellular matrix to the cytoskeleton. The laminin-binding integrin $\alpha 3 \beta 1$ is expressed at high levels in lung epithelium and in kidney podocytes. In podocytes, $\alpha 3 \beta 1$ associates with the tetraspanin CD151 to maintain a functional filtration barrier. Here, we report on a patient homozygous for a novel missense mutation in the human ITGA3 gene, causing fatal interstitial lung disease and congenital nephrotic syndrome. The mutation caused an alanine-to-serine substitution in the integrin $\alpha 3$ subunit, thereby introducing an $\mathrm{N}$-glycosylation motif at amino acid position 349 . We expressed this mutant form of ITGA3 in murine podocytes and found that hyperglycosylation of the $\alpha 3$ precursor prevented its heterodimerization with $\beta 1$, whereas $\mathrm{CD} 151$ association with the $\alpha 3$ subunit occurred normally. Consequently, the $\beta 1$ precursor accumulated in the ER, and the mutant $\alpha 3$ precursor was degraded by the ubiquitin-proteasome system. Thus, these findings uncover a gain-of-glycosylation mutation in ITGA3 that prevents the biosynthesis of functional $\alpha 3 \beta 1$, causing a fatal multiorgan disorder.
\end{abstract}

\section{Introduction}

The integrin family is comprised of 24 transmembrane heterodimeric $\alpha \beta$ glycoproteins that link the extracellular matrix to the cytoskeleton (1). Most integrins connect to actin filaments and reside in cellular adhesion structures designated focal adhesions (FAs), which are highly enriched in tyrosine-phosphorylated proteins and serve as major hubs for signal transduction (2). Integrin-ligand binding can be controlled by conformational changes that tune integrin affinity (3). Furthermore, integrin function depends strongly on trafficking events, which include endocytosis, intracellular sorting and recycling, and delivery of de novo synthesized integrins to the plasma membrane by the biosynthetic route $(4,5)$. Both $\alpha$ and $\beta$ subunits are synthesized as precursors. After $N$-linked glycosylation, folding, and association of the $\alpha$ and $\beta$ subunits in the ER, the heterodimer is transported to the Golgi network, in which the $N$-linked high-mannose oligosaccharides are further processed into complex oligosaccharides. Subsequently, several of the $\alpha$ subunit precursors, including $\alpha 3$, are cleaved by proprotein convertases such as furin into a heavy and a light chain, which are held together by a disulphide bond to generate the mature form that is expressed at the plasma membrane $(6,7)$.

A number of human congenital disorders have been associated with defective integrin-mediated adhesion, including the blistering disorder epidermolysis bullosa (integrin $\alpha 6 \beta 4$ in epithelia), the bleeding disorder Glanzmann's thrombasthenia (integrin $\alpha \operatorname{IIb} \beta 3$ on platelets), leukocyte adhesion deficiency-I ( $\beta 2$ integrins on leukocytes), and muscular dystrophy (integrin

Authorship note: Nayia Nicolaou and Coert Margadant, as well as Kirsten Y. Renkema and Arnoud Sonnenberg, contributed equally to this work. Conflict of interest: The authors have declared that no conflict of interest exists. Citation for this article: J Clin Invest. 2012;122(12):4375-4387. doi:10.1172/JCI64100. $\alpha 7 \beta 1$ in striated muscle) (8-11). Furthermore, the importance of integrins for cell adhesion in a variety of organ systems has been demonstrated by numerous mouse models targeting either integrin subunits or integrin-associated proteins (12). For example, genetic deletion of the laminin-binding (LN-binding) epithelial integrin $\alpha 3 \beta 1$ in mice causes severe lung and kidney defects as well as abnormalities in the epidermis (13-17). In podocytes, which are the epithelial cells of the glomerulus of the kidney, $\alpha 3 \beta 1$ maintains cell adhesion under great dynamic stress by binding to LN-511/521 in the glomerular basement membrane (GBM). Integrin-LN binding is reinforced by intracellular connection to the actin cytoskeleton via linker proteins, such as $\alpha$-actinin or integrin-linked kinase, and by lateral association with the tetraspanin CD151 (18). The pivotal role of this multicomponent adhesion unit in regulating the barrier function of the kidney is illustrated by the severe glomerular defects observed in mice upon (podocyte-specific) deletion of $\alpha 3, \beta 1$, integrin-linked kinase, CD151, or the $\alpha 5$ chain of LN-511/521 $(19-25)$. In addition, mutations in the genes encoding the $\beta 2$ chain of LN-521, $\alpha$-actinin-4, and CD151 have been identified in patients suffering from renal disorders, including Pierson syndrome, focal segmental glomerulosclerosis, and hereditary nephritis (26-28).

Here, we report a mutation in the human ITGA3 gene in a patient with interstitial lung disease and congenital nephrotic syndrome. The mutation leads to a gain of glycosylation, which impedes heterodimerization of the $\alpha 3$ precursor with $\beta 1$ but not its association with CD151. As a consequence, the $\beta 1$ precursor accumulates in the ER, and the mutant $\alpha 3$ precursor is cleared by ubiquitination and degradation by the proteasome. Thus, we have identified a gain-of-glycosylation mutation in ITGA3 that prevents the biosynthesis of functional $\alpha 3 \beta 1$, leading to severe kidney and lung defects. 
A
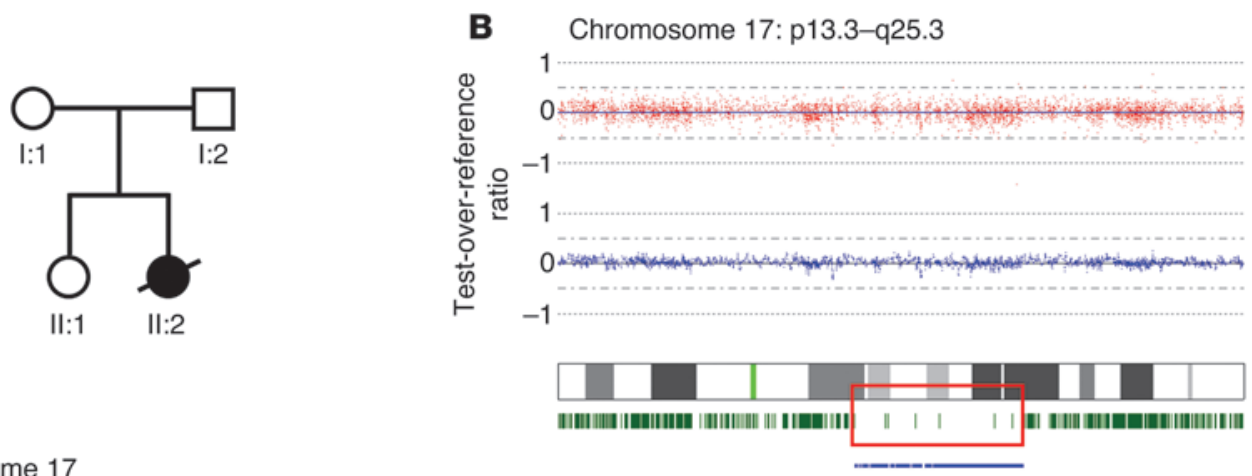

C Chromosome 17

\begin{tabular}{|l|l|l|l|l|l|l|l|l|l|l|l|l|l|l|l|l|l|}
\hline p13.3p13.2 p13.1 & $p 12$ & $p 11.2$ & $q 11.2$ & $q 12$ & $\mid q 21.2$ & $q 21.31$ & 21.32 & $q 21.33$ & $q 22$ & $q 23.2$ & 23.324 .1 & $q 24.2$ & $q 24.3$ & $q 25.1$ & $q 25.3$ \\
\hline
\end{tabular}

D
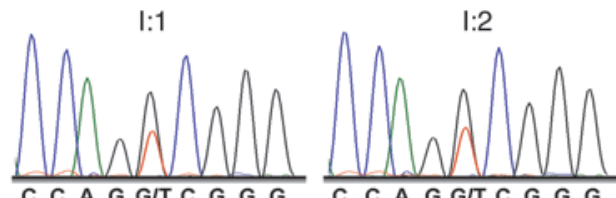

II:1

II:2

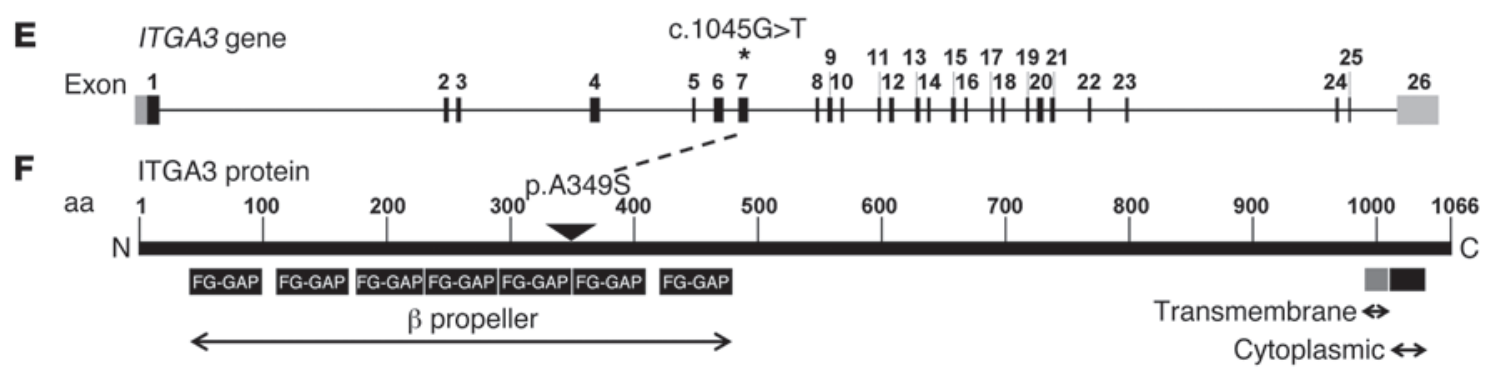

Figure 1

Identification of homozygosity for an ITGA3 mutation in a patient with interstitial lung disease and nephrotic syndrome. (A) Pedigree of the patient's family. I:1 and I:2, the patient's parents; II:2, the patient; II:1, the patient's sister. (B) Results from the 250k SNP array performed on the patient's DNA. $\log _{2}$ test-over-reference ratio values ( $y$ axis) for SNP loci plotted against the position on chromosome 17 ( $x$ axis). Red dots represent the $\log _{2}$ ratio for each SNP locus (top panel). The effective hidden Markov model outcome, with a normal test-over-reference ratio of 0 . Blue dots represent the mean $\log _{2}$ ratio of neighboring SNPs on the array, indicating no significant copy number gains or losses (middle panel). Idiogram of chromosome 17. Green marks represent heterozygous SNP calls at particular DNA loci. The red box indicates the homozygous 19.2-Mb region, reflected by a loss of heterozygous loci. The thickness of the blue line represents the likelihood of LOH (bottom panel). (C) Idiogram of chromosome 17. The homozygous region from 17q12 to $17 q 23.2$ is boxed. (D) Sequence analysis of ITGA3 in the patient's parents, the patient, and the patient's sister. The patient is homozygous for a missense mutation (c.1045G>T), resulting in the amino acid substitution p.A349S. Both the parents and the sister are heterozygous for the mutation. (E) Schematic overview of the 26 exons of the ITGA3 gene. The location of the c. $1045 \mathrm{G}>\mathrm{T}$ mutation in exon 7 is indicated by an asterisk. (F) Domain organization of the integrin $\alpha 3$ subunit. The A349S mutation is located between 2 FG-GAP repeats in the extracellular $\beta$-propeller domain.

\section{Results}

A point mutation in ITGA3 causes congenital nephrotic syndrome and interstitial lung disease. We identified a patient born prematurely, at a gestational age of 36 weeks, to unaffected nonconsanguineous Dutch parents. She had one unaffected sibling (Figure 1A). At birth, the patient presented with respiratory distress. The electrocardiogram was normal, and no structural heart defects were observed by ultrasound. However, lung biopsies revealed interstitial lung disease. In addition, renal ultrasound revealed unilateral kidney hypoplasia with hydronephrosis on the left side, and the patient was diagnosed with congenital nephrotic syndrome. Growth retardation was prominent, which could in part be attributed to prolonged steroid treatment and intermittent feeding difficulties. The patient died at the age of 7 months due to respiratory insufficiency. We first screened known genes implicated in nephrotic syndrome (NPHS1, NPHS2, PLCE1, WT1, LAMB2, TRPC6, ACTN4, and INF2) and surfactant metabolism dysfunction, which is implicated in perinatal respiratory distress $(A B C A 3, S F T P B$, and SFTPC). No mutations were detected in these genes (K.Y. Renkema, unpublished observations). Subsequently, we conducted genome-wide screening in the patient and both parents for copy number variations (CNVs), using Affymetrix 250K SNP array analysis. The data have been deposited in NCBI's Gene Expression Omnibus and are accessible through GEO Series accession number GSE40405 (http://www.ncbi.nlm. nih.gov/geo/query/acc.cgi?acc=GSE40405). Whereas no clinically relevant $\mathrm{CNVs}$ were detected, a 19.2-Mb long continuous stretch of homozygosity (36.78-55.97 Mb) was identified on chromosome 17, encompassing 1,261 SNP probes, with SNP_A_2105989 and SNP_A_4223473 as bordering SNPs (Figure 1, B and C). The 
A

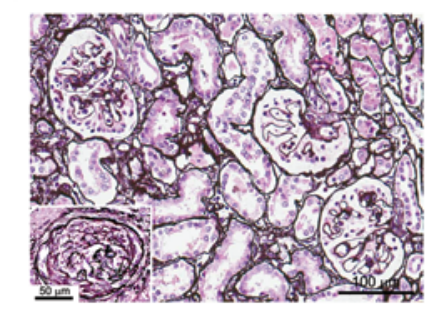

B

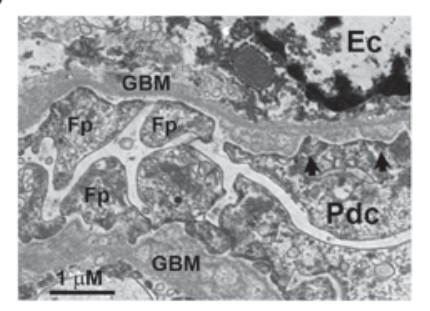

C
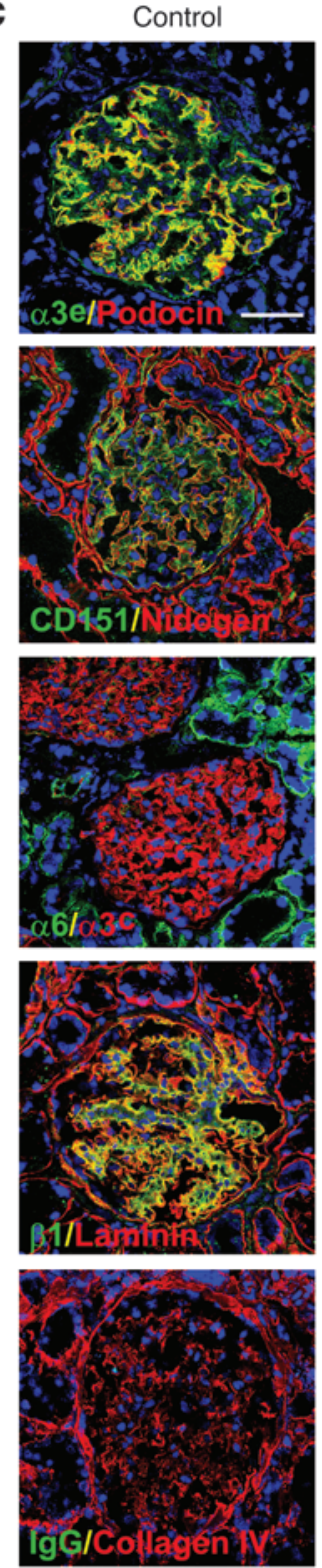

Patient
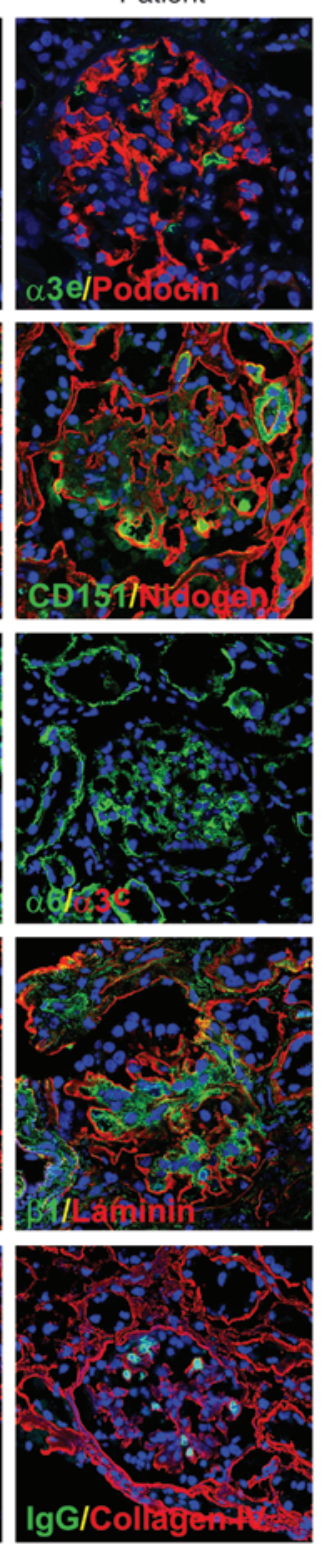

\section{Figure 2}

Abnormalities in the kidneys of the patient. (A) Jones' silver staining of a section of patient kidney, showing a highly irregular mesangium and fewer and wider capillary loops, with highly variable numbers of podocytes. The inset shows a fibrocellular crescent in a glomerulus undergoing glomerulosclerosis. Scale bar: $100 \mu \mathrm{m} ; 50 \mu \mathrm{m}$ (inset). (B) Ultrastructural analysis of the filtration barrier of the kidney of the patient, demonstrating abnormal foot processes and thickening of the GBM with local protrusions. Ec, endothelial cell; Fp, foot processes; Pdc, podocyte. Scale bar: $1 \mu \mathrm{m}$. (C) Kidney cryosections were subjected to indirect immunofluorescence analysis. The images show glomeruli stained with antibodies against the extracellular domain of $\alpha 3^{\mathrm{e}}$ (J143; green) and podocin (red), CD151 (green) and nidogen (red), $\alpha 6$ (green) and the cytoplasmic domain of $\alpha 3\left(\alpha 3^{c}\right)$ (homemade polyclonal antibody; red), $\beta 1$ (green) and LN (red), and control IgG (green) and collagen IV (red). Nuclei were counterstained with DAPI (blue). Scale bar: $50 \mu \mathrm{m}$. region contained 566 genes, among which was the ITGA3 gene, which encodes the integrin $\alpha 3$ subunit. The possibility of uniparental disomy of chromosome 17 was excluded by investigating the Mendelian inheritance pattern of 4,854 SNPs that are located on chromosome 17 and which are present on the array (K.Y. Renkema, unpublished observations). We then directly sequenced the ITGA3 coding sequence, which revealed homozygosity for a missense variant c.1045G $>\mathrm{T}$, for which both parents and the healthy sibling were heterozygous (Figure 1D). In contrast, the variant was not observed in 384 chromosomes of ethnically matched (Dutch) individuals. In addition, it has not been annotated in dbSNP, the NHLBI Exome Sequencing Project, or our in-house database of 100 exome-sequencing projects. The ITGA3 gene extends over $34.5 \mathrm{~kb}$ and contains 26 exons. The identified mutation is located in exon 7 (Figure 1E). At the protein level, the mutation causes the substitution of alanine 349 for serine (A349S) in an extracellular domain of $\alpha 3$ designated the $\beta$-propeller (Figure 1F). In summary, we have identified a missense mutation in ITGA3 in a patient with interstitial lung disease and congenital nephrotic syndrome.

The A349S mutation prevents integrin $\alpha 3 \beta 1$ expression in vivo and causes severe kidney and lung abnormalities. Congenital nephrotic syndrome is characterized by proteinuria, resulting from defects in the filtration barrier of the glomerulus. To characterize the defects that caused the congenital nephrotic syndrome in our patient, we performed histological examination of kidney biopsies. Severe abnormalities were observed, including aberrant glomerular morphology and cystic dilation of tubuli and local protein casts as well as interstitial fibrosis and some inflammatory infiltrate. The GBM was irregularly thickened, and the glomeruli consisted of fewer and wider capillary loops covered by highly variable numbers of podocytes. In addition, mesangiolysis and mesangial hypercellularity, occasional extracapillary proliferation reflecting a loss of capillary integrity, and focal segmental glomerulosclerosis were observed 

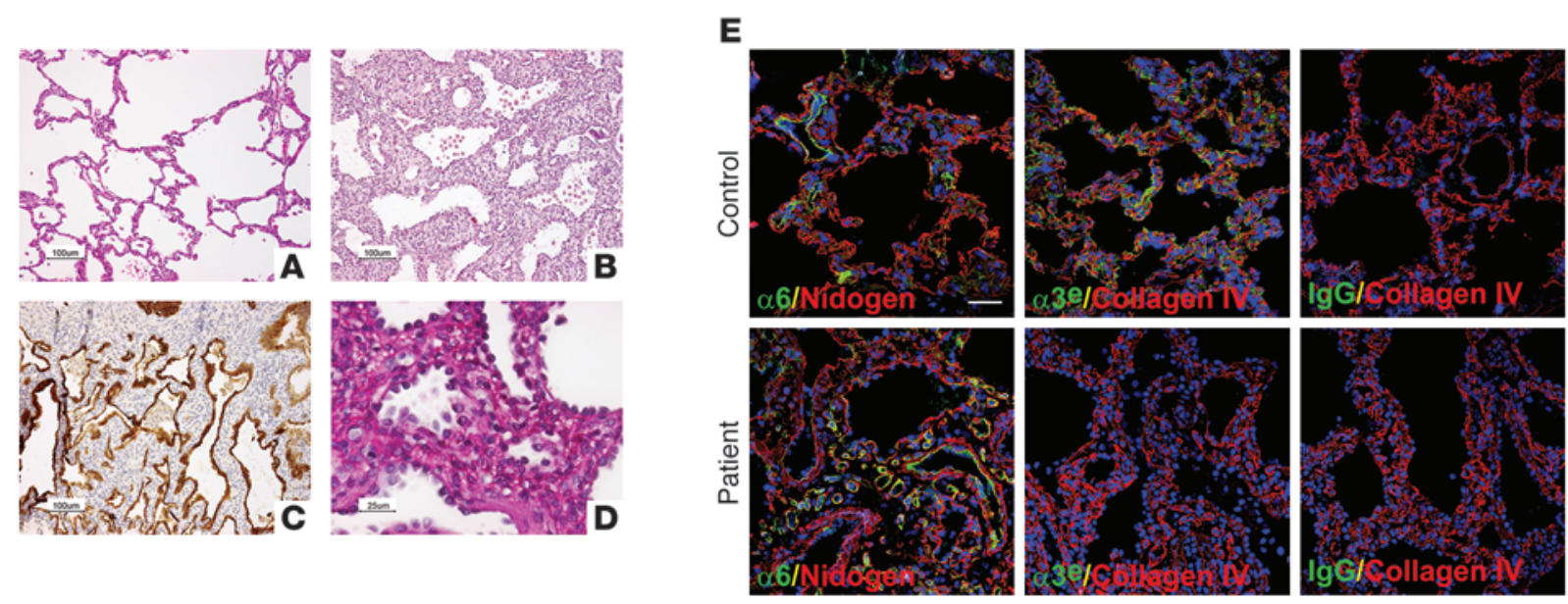

\section{Figure 3}

Abnormalities in lung tissue of the patient. (A) H\&E staining of a lung section from a healthy individual. Scale bar: $100 \mu \mathrm{m}$. (B) H\&E staining of a lung section from the patient. Scale bar: $100 \mu \mathrm{m}$. (C) Keratin staining showing lining of the thickened alveolar septa with reactive type II pneumocytes in patient lungs. Scale bar: $100 \mu \mathrm{m}$. (D) PAS staining demonstrating glycogen deposits in interstitial cells in the lungs of the patient. Scale bar: $25 \mu \mathrm{m}$. (E) Lung cryosections of the patient and a control were subjected to indirect immunofluorescence analysis using our homemade antibodies against the cytoplasmic tail of $\alpha 3$ (green) and collagen IV (red) or $\alpha 6$ (green) and nidogen (red). IgG was included as a control for antibody specificity. Nuclei were counterstained with DAPI (blue). Scale bar: $50 \mu \mathrm{m}$.

(R. Goldschmeding, unpublished observations; Figure 2A; and Supplemental Figure 1; supplemental material available online with this article; doi:10.1172/JCI64100DS1). Ultrastructural analysis revealed partial effacement of foot processes and a disorganized and thickened GBM, displaying a multilayered and pseudo-reticular structure with loose fragments, suggestive of a disturbance of the fusion process (Figure 2B and R. Goldschmeding, unpublished observations).

We then investigated the expression and distribution of individual glomerular components by immunofluorescence analysis of kidney cryosections. In the patient's glomeruli, the integrin $\alpha 3$ subunit could not be detected, neither with antibodies J143 or P1B5, which are directed against the extracellular domain of $\alpha 3$, nor with a homemade antibody that recognizes the cytoplasmic tail (Figure 2C and A. Sonnenberg and R. Goldschmeding, unpublished observations). Some aspecific immunoreactivity was observed in focal patches of cells. These also reacted with control IgG and most likely consist of activated mesangial cells, which are known to express Fc receptors. In addition to the lack of $\alpha 3$, a dramatic decrease in the levels of the $\beta 1$ subunit was visible, confirming that $\alpha 3 \beta 1$ is the main $\beta 1$ integrin in the glomerulus. Also, the expression of podocin and CD151 seemed reduced in the glomeruli of the patient, whereas expression of the integrin $\alpha 6$ subunit appeared to be slightly increased. Furthermore, there was an increase in the expression and deposition of the GBM proteins LN, nidogen, and collagen IV, confirming the thickening of the GBM observed by electron microscopy (Figure 2C).

Next, we analyzed lung biopsies from the patient. There were also multiple abnormalities in the lung, including widened alveolar septa lined with reactive type II pneumocytes and increased numbers of alveolar macrophages, whereas the alveolar walls in an agematched healthy individual were lined with flat type I pneumocytes and contained virtually no reactive type II pneumocytes and no alveolar macrophages (Figure 3, A-C). Pronounced pulmonary interstitial glycogenesis was detected by PAS staining (Figure 3D). In addi- tion, we observed an abnormal deposition of surfactant by electron microscopy (R. Goldschmeding, unpublished observations). As in the kidney, $\alpha 3$ could not be detected by immunofluorescence analysis of lung cryosections, whereas the expression of $\alpha 6$ seemed to be increased (Figure 3E). Together, these data show that the A349S mutation leads to the lack of integrin $\alpha 3 \beta 1$ expression, causing severe abnormalities in the kidneys and the lungs.

The A349S mutation impairs the expression of mature $\alpha 3$ at the cell surface. To investigate the effect of the A349S mutation in vitro, we isolated glomeruli from Itga $3^{f l / f l} \mathrm{Cd} 151^{f l / f l} \mathrm{Trp} 53^{+/-}$mice, according to the Dynabead perfusion method, as described previously (29). Outgrowing epithelial cells were sorted for the expression of podocalyxin and the absence of the endothelial cell marker CD31. The floxed Itga3 and Cd151 genes were deleted by adenoviral delivery of Cre-recombinase, and the absence of both proteins was confirmed by flow cytometry (Supplemental Figure 2). We then generated the A349S mutation in cDNA encoding human $\alpha 3$ and stably expressed either wild-type human $\alpha 3\left(\alpha 3^{\mathrm{WT}}\right)$ or mutant human $\alpha 3\left(\alpha 3^{\mathrm{A} 349 \mathrm{~S}}\right)$ in the $\alpha 3 / \mathrm{CD} 151$-deficient podocytes by retroviral transduction, followed by selection with zeocin. The podocytes were also reconstituted with FLAG-tagged human CD151 to avoid possible differences in binding efficiency of murine CD151 to human $\alpha 3$, because of sequence variability in the $\alpha 3$-binding QRD motif, which is KRD in the mouse (30).

We first determined the cell surface expression of various integrin subunits and CD 151 by flow cytometry. In line with the results of immunofluorescence analysis of the patient tissue, $\alpha 3$ was completely absent from the cell surface of podocytes expressing $\alpha 3^{\mathrm{A} 349 \mathrm{~S}}$, and $\beta 1$ levels were strongly reduced (Figure $4 \mathrm{~A}$ ). In contrast, the cell surface expression of CD151, as well as that of the integrin subunits $\alpha 2, \alpha 5$, and $\alpha 6$, was similar in podocytes expressing $\alpha 3^{\mathrm{WT}}$ or $\alpha 3^{\mathrm{A} 349 \mathrm{~S}}$.

Next, we analyzed the expression of precursor and mature $\alpha 3$ by Western blotting. The $\alpha 3$ subunit is synthesized as a precursor of approximately $150 \mathrm{kDa}$. After $N$-linked glycosylation in the 
A
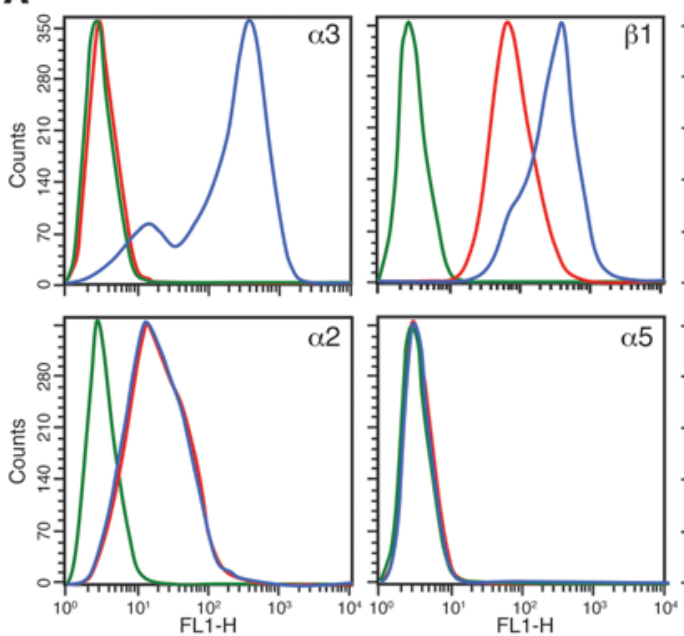

$\alpha 5$
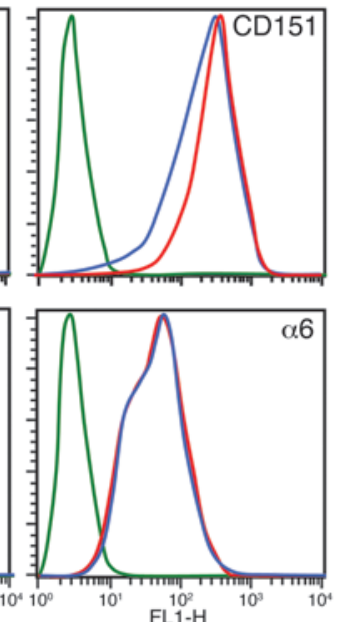

- Podocytes $\left(\mathrm{CD}_{151^{+}}, \alpha 3^{-}\right)$, negative IgG control

- Podocytes (CD151*, $\alpha 3^{\mathrm{WT})}$

- Podocytes $\left(\mathrm{CD} 151^{*}, \alpha 3^{\text {3as95 }}\right)$
B

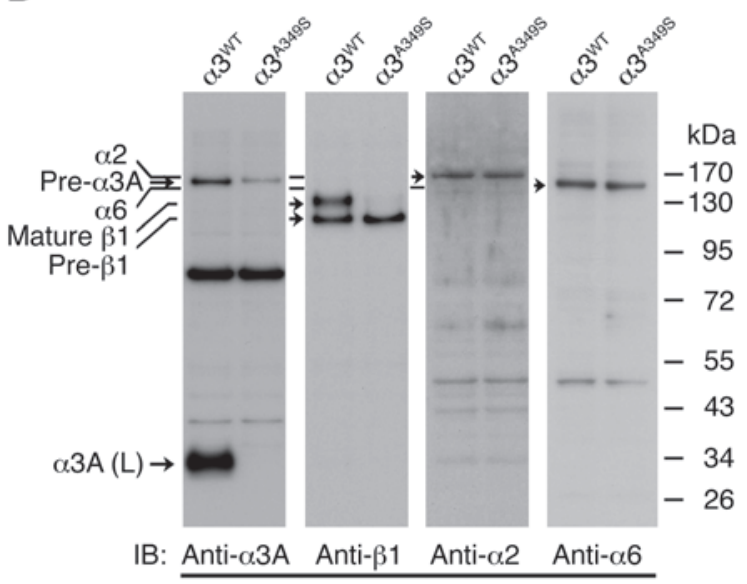

WCL

Figure 4

The $\alpha 3^{\text {A349S }}$ mutation prevents expression of mature but not precursor $\alpha 3$ in podocytes. (A) Murine $\alpha 3 / C D 151$-deficient podocytes were retrovirally transduced with human CD151 as well as with either human wild-type $\alpha 3$ (blue) or human $\alpha 3$ carrying the patient mutation (red), and positive cells were selected with zeocin. Cell surface expression of integrin subunits $\alpha 2, \alpha 3, \alpha 5, \alpha 6$, and $\beta 1$ as well as CD151 was determined by flow cytometry. Green indicates cells incubated only with secondary antibody. (B) Expression of the $\alpha 3$ subunit (precursor [ 150 kDa] and mature light (L) chain $[\sim 35 \mathrm{kDa}]$ ) as well as that of the $\beta 1, \alpha 2$, and $\alpha 6$ subunits was investigated by Western blotting in whole-cell lysates (WCLs) of podocytes. Arrows in the second column indicate the mature $\beta 1$ (top arrow) and precursor $\beta 1$ (bottom arrow); arrows in the third and fourth columns indicate $\alpha 2$ and $\alpha 6$, respectively.

ER and association with $\beta 1$, the $\alpha 3 \beta 1$ heterodimer is transported to the Golgi. Here, processing of the $N$-linked oligosaccharides occurs, and the $\alpha 3$ precursor is cleaved into a heavy $(\sim 115 \mathrm{kDa})$ and a light chain $(-35 \mathrm{kDa})(6,7)$. The $\beta 1$ subunit is synthesized as a partially glycosylated precursor of approximately $105 \mathrm{kDa}$ and a more heavily glycosylated mature form of approximately $125 \mathrm{kDa}$ that is expressed at the cell surface. Intriguingly, we clearly detected precursor $\alpha 3^{\mathrm{A} 349 \mathrm{~S}}$, though to a lesser extent than $\alpha 3^{\mathrm{WT}}$, suggesting that the mutant is either less expressed or that some degradation occurs. The mature product was nevertheless completely absent, as evidenced by the lack of the $\alpha 3$ light chain (Figure 4B). Consistently, there was a large pool of $\beta 1$ precursor but a dramatic reduction of mature $\beta 1$, as compared with the level in podocytes expressing wild-type $\alpha 3$. This observation supports the results obtained by flow cytometry and suggests that the mutant $\alpha 3$ precursor fails to heterodimerize, leading to accumulation of $\beta 1$ precursor and a strong reduction of mature $\beta 1$. These data indicate that, whereas the A349S mutation does not impair the expression of precursor $\alpha 3$, it leads to a lack of the mature $\alpha 3$ subunit at the cell surface, probably because heterodimerization with $\beta 1$ is prevented.

The A349S mutation impairs $\alpha 3 \beta 1$ heterodimerization but not the association of precursor $\alpha 3$ with CD151. To determine whether the A349S mutation indeed prevents heterodimerization of the $\alpha 3$ and $\beta 1$ subunits, we immunoprecipitated $\alpha 3$ from lysates of podocytes expressing $\alpha 3^{\mathrm{WT}}$ or $\alpha 3^{\mathrm{A} 349 \mathrm{~S}}$. The $\alpha 3$ subunit was precipitated with either antibodies against its cytoplasmic tail (29A3) or antibodies that recognize its extracellular domain (J143 and P1B5), whereas detection of $\alpha 3$ was performed using our homemade antibodies directed against its cytoplasmic tail. On reducing gels, this antibody recognizes the precursor of $\alpha 3$ as well as the $35-\mathrm{kDa}$ light chain of the mature $\alpha 3$ subunit. In lysates from podocytes expressing $\alpha 3^{\mathrm{WT}}$, all antibodies precipitated both mature $\alpha 3$ and the precursor, although the latter was recognized to a lesser extent by $\mathrm{J} 143$ and P1B5 than by 29A3. Precursor $\alpha 3^{\mathrm{WT}}$ appeared as 2 bands, which likely represent the high-mannose form in the ER and the complex form after modification of the $N$-linked oligosaccharides in the Golgi apparatus. As expected, the $\beta 1$ subunit was coprecipitated with $\alpha 3^{\mathrm{WT}}$. In contrast, neither J143 nor P1B5 precipitated the $\alpha 3^{\mathrm{A} 349 \mathrm{~S}}$ precursor, whereas $29 \mathrm{~A} 3$ did precipitate precursor $\alpha 3^{\mathrm{A} 349 \mathrm{~S}}$ but not the mature $\alpha 3$ or $\beta 1$ subunits (Figure 5A). These results suggest that the $\alpha 3^{\mathrm{A} 349 \mathrm{~S}}$ precursor does not associate with $\beta 1$ and that J143 and P1B5 recognize a complex-dependent epitope on the $\alpha 3$ subunit. We then precipitated the $\beta 1$ subunit from podocyte lysates, using either the MB1.2 or the 9EG7 antibodies. Intriguingly, 9EG7 almost exclusively precipitated the $\beta 1$ precursor but little or no mature $\beta 1$, whereas MB1.2 precipitated both the precursor and mature $\beta 1$, together with mature $\alpha 3^{\mathrm{WT}}$. In contrast, precursor $\beta 1$, but hardly any mature $\beta 1$, was precipitated from lysates of podocytes expressing the mutant $\alpha 3$ subunit (Figure 5B). Furthermore, neither of the 2 antibodies against $\beta 1$ coprecipitated $\alpha 3^{\mathrm{A} 349 \mathrm{~S}}$. These results confirm that the A349S mutation prevents association of the $\alpha 3$ subunit with $\beta 1$ in cultured podocytes. To analyze whether heterodimerization was also impaired in the patient, we precipitated $\alpha 3$ with 29A 3 from a lysate of patient lung tissue. Consistent with the observations in podocytes, both precursor and mature $\alpha 3$, as well as $\beta 1$, were coprecipitated with 29A3 from tissue of an unaffected individual, whereas no mature $\alpha 3$ or $\beta 1$ and only a small amount of precursor $\alpha 3$ was precipitated from patient tissue (Figure 5C). We then investigated the association of $\alpha 3^{\mathrm{WT}}$ and $\alpha 3^{\mathrm{A} 349 \mathrm{~S}}$ with CD151 in podocytes. For this purpose, 
A

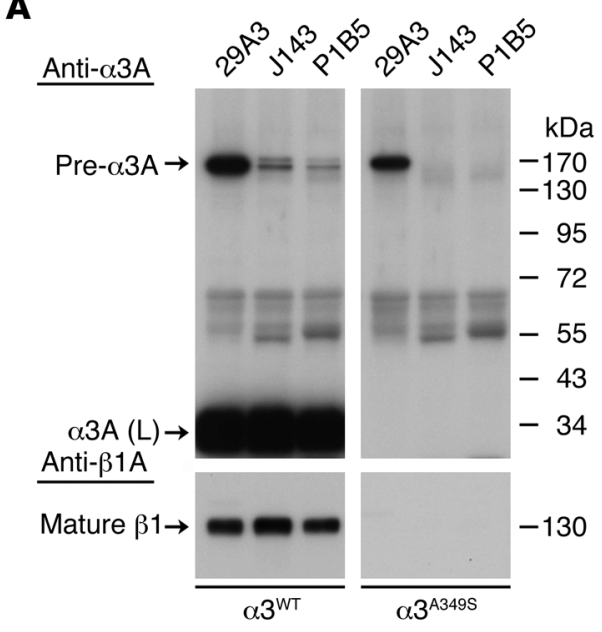

C

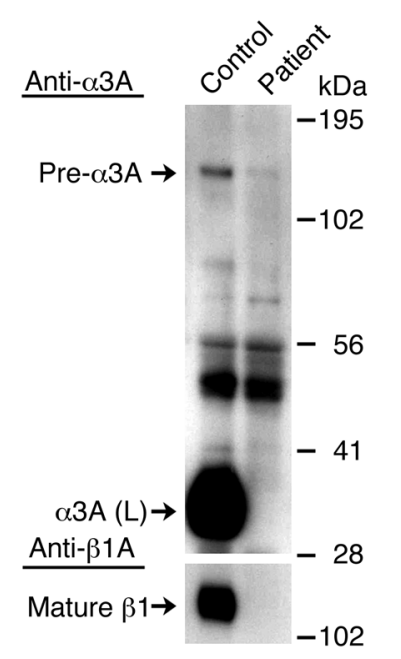

B

D
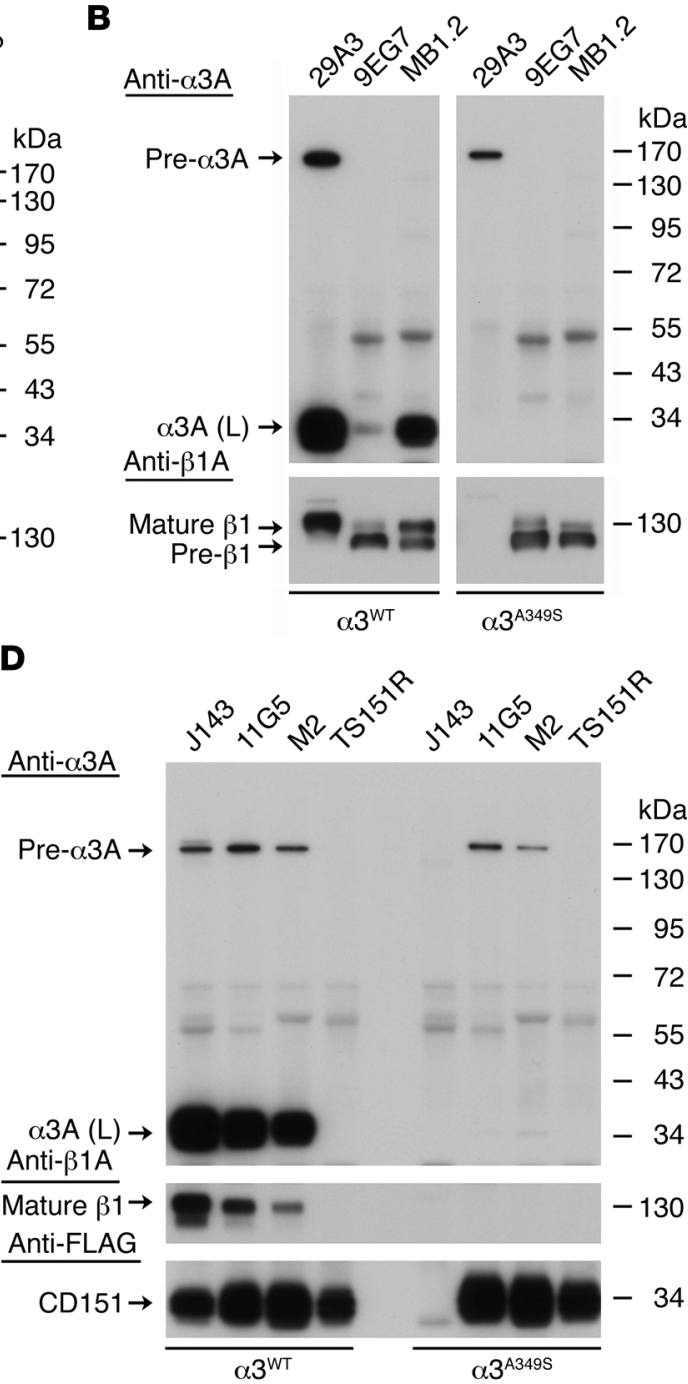

\section{Figure 5}

The $\alpha 3^{\text {A349S }}$ mutation disrupts $\alpha 3$ heterodimerization with $\beta 1$ but not CD151 binding. (A) Podocytes expressing $\alpha 3^{\mathrm{WT}}$ or $\alpha 3^{\mathrm{A} 349 \mathrm{~S}}$ were lysed, and the $\alpha 3$ subunit was precipitated using antibodies against the cytoplasmic tail (29A3) or the extracellular domain (J143 and P1B5). Precipitated $\alpha 3$ and $\beta 1$ were detected by Western blotting. (B) $\beta 1$ was precipitated from podocyte lysates using 9EG7 or MB1.2, and $\alpha 3$ and $\beta 1$ were detected by Western blotting. (C) The $\alpha 3$ subunit was precipitated using $29 \mathrm{~A} 3$ from tissue lysates of the patient and a healthy individual, and $\alpha 3$ and $\beta 1$ were detected by Western blotting. (D) FLAG-tagged CD151 was precipitated from podocyte lysates using M2 against the FLAG-tag, 11G5 against CD151, or TS151R that recognizes the QRD sequence in CD151 when not in complex with $\alpha 3$. J143 was included as a control. CD151, $\alpha 3$, and $\beta 1$ were subsequently detected by Western blotting. we performed immunoprecipitation experiments using either the 11G5 antibodies against CD151 or M2, which is directed against the FLAG-tag. As a negative control, we included the TS151R antibodies that recognize the QRD sequence in CD151 that interacts with $\alpha 3$ and thus can only bind to CD151 when not in complex with $\alpha 3 \beta 1(31,32)$. Both M2 and 11 G5 coprecipitated CD151 with precursor and mature $\alpha 3^{\mathrm{WT}}$ as well as with $\beta 1$, suggesting that CD151 can associate both with the $\alpha 3$ precursor and mature $\alpha 3$. In addition, both antibodies clearly coprecipitated CD151 with $\alpha 3^{\mathrm{A349S}}$ but not with $\beta 1$, indicating that association of CD 151 with the mutant precursor is not impaired and that CD151- $\alpha 3$ association can occur prior to the association of $\alpha 3$ with $\beta 1$ (Figure 5D). As expected, the TS151R antibodies only precipitated CD151 and no integrin subunits. These data suggest that CD151 can associate with precursor $\alpha 3$ prior to $\alpha / \beta$ heterodimerization and that the A349S mutation prevents $\alpha 3$ heterodimerization with $\beta 1$ but not its association with CD151.

Gain of glycosylation disrupts heterodimerization and cell surface expression. To understand the mechanism by which the A349S mutation impairs $\alpha / \beta$ heterodimerization, we first determined the position of alanine 349 in the 3 -dimensional structure of $\alpha 3 \beta 1$ by homology modeling based on the recently resolved crystal structure of $\alpha 5 \beta 1$ (33). Alanine 349 is located on a loop opposite to the $\alpha / \beta$ interface, suggesting that a mutation at this site does not directly impair $\alpha 3 \beta 1$ heterodimerization by steric hindrance (Figure 6A). The A349S substitution introduces a novel $N$-glycosylation motif in $\alpha 3(\mathrm{~N}-\mathrm{x}-\mathrm{A}>\mathrm{N}-\mathrm{x}-\mathrm{S})$, indicating that it leads to a gain of glycosylation. This was further suggested by the reduced mobility of the $\alpha 3^{\mathrm{A} 349 \mathrm{~S}}$ precursor in gel electrophoresis on low-percentage gels, whereas both the $\alpha 3^{\mathrm{WT}}$ and the $\alpha 3^{\mathrm{A} 349 \mathrm{~S}}$ precursor migrated with similar mobility after incubation of the precipitated proteins with $N$-glycanase, an enzyme that cleaves all $N$-linked high-mannose oligosaccharides (Figure 6B). Alternatively, treatment of cells with tunicamycin, a pharmacological inhibitor of $N$-linked glycosylation, also abolished the difference in mobility between $\alpha 3^{\mathrm{WT}}$ and $\alpha 3^{\mathrm{A} 349 \mathrm{~S}}$ precursors, confirming that $\alpha 3^{\mathrm{A} 349 \mathrm{~S}}$ was indeed hyperglycosylated (Figure 6C). However, the removal of all $N$-linked sugars by tunicamycin did not rescue heterodimerization but instead induced degradation of both the $\alpha 3$ and $\beta 1$ subunits, reflecting the essential role of $N$-glycosylation in protein stability. To demonstrate that the addition of a sugar moiety prevents $\alpha / \beta$ heterodimerization, alanine 349 in $\alpha 3$ was substituted for glycine $\left(\alpha 3^{\mathrm{A} 349 \mathrm{G}}\right)$, which cannot be glycosylated, and the $\alpha 3^{\mathrm{A} 349 \mathrm{G}}$ mutant was introduced in $\alpha 3 /$ CD151-deficient podocytes, as described above. 
A
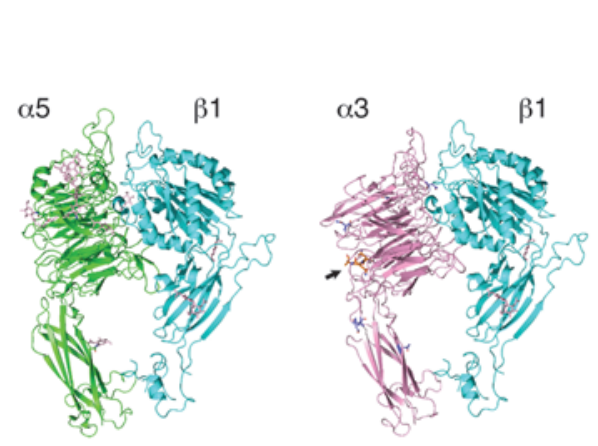

B

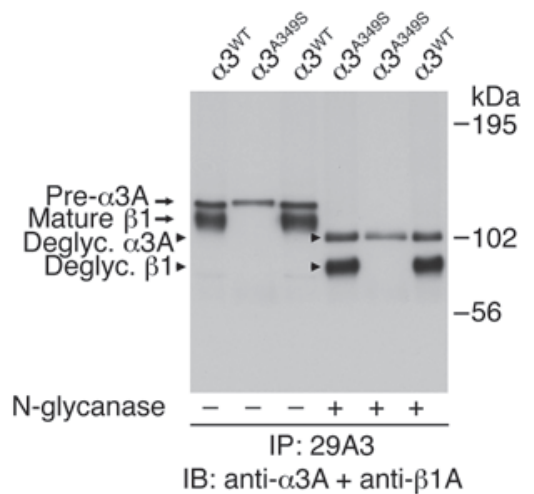

IB: anti- $\alpha 3 A+$ anti- $\beta 1 A$

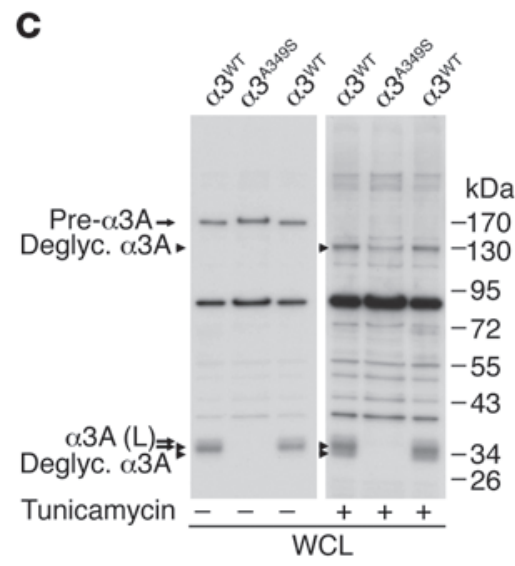

IB: anti- $\alpha 3 \mathrm{~A}$
D

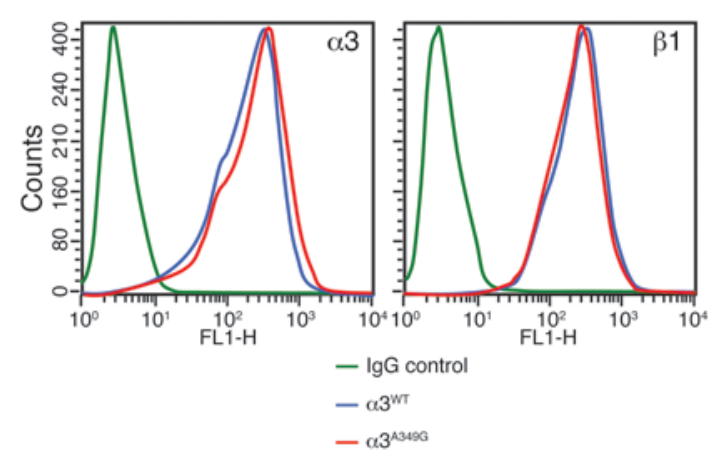

E

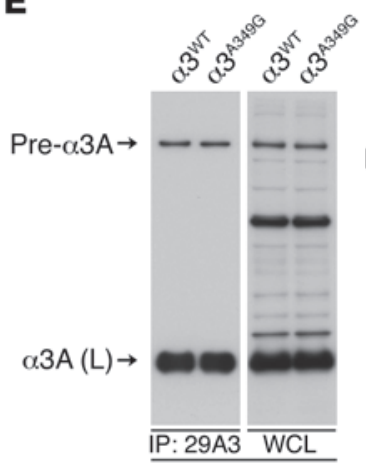

IB: anti- $\alpha 3 \mathrm{~A}$

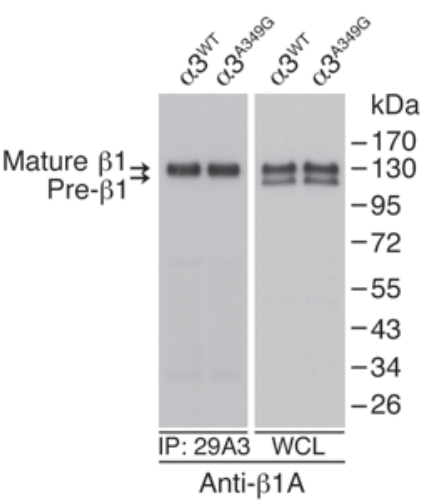

\section{Figure 6}

The $\alpha 3^{\text {A349S }}$ mutation introduces a glycosylation motif that impairs heterodimerization and cell surface expression. (A) Model of the ectodomain of $\alpha 5 \beta 1$ and $\alpha 3 \beta 1$. The $\mathrm{N}-\mathrm{x}$-A motif is indicated by an arrow. (B) The $\alpha 3$ subunit was precipitated with $29 \mathrm{~A} 3$, the precipitates were treated with $\mathrm{N}$-glycanase, and $\alpha 3$ and $\beta 1$ were detected by Western blotting. (C) Podocytes expressing either $\alpha 3^{W T}$ or $\alpha 3^{\text {A349S }}$ were cultured for 16 hours in the absence or the presence of tunicamycin $(1 \mu \mathrm{g} / \mathrm{ml})$, after which expression of the $\alpha 3$ subunit was analyzed by Western blotting. (D) Alanine 349 was mutated to glycine in human $\alpha 3$, and the $\alpha 3^{\mathrm{A} 349 \mathrm{G}}$ mutant was expressed in $\alpha 3$-deficient podocytes by retroviral transduction and selection with zeocin. Cell surface expression of $\alpha 3$ and $\beta 1$ in $\alpha 3^{W T}$-expressing (blue) or $\alpha 3^{A 349 G}$-expressing (red) cells was then investigated by flow cytometry. Green indicates cells incubated only with secondary antibody. (E) Expression of precursor and mature $\alpha 3^{A 349 G}$ as well as heterodimerization with $\beta 1$ were investigated by precipitation of $\alpha 3$ with antibody 29A3 from lysates of podocytes expressing either $\alpha 3^{W T}$ or $\alpha 3^{A 349 G}$, followed by Western blotting.

Intriguingly, flow cytometry indicated that $\alpha 3^{\mathrm{A} 349 \mathrm{G}}$ was expressed at normal levels on the cell surface (Figure 6D). Consistent with this result, immunoprecipitation followed by Western blotting demonstrated that the $\alpha 3^{\mathrm{A} 349 \mathrm{G}}$ precursor is normally cleaved to generate the mature product and that $\alpha 3^{\mathrm{A} 349 \mathrm{G}}$ indeed associates with the $\beta 1$ subunit (Figure 6E). Thus, whereas the A349S substitution has dramatic consequences, substitution of alanine 349 with glycine has no effect. In line with this result, alignment of the amino acid sequence of $\alpha 3$ from different species revealed that a glycine, as well as an arginine or asparagine but not a serine or threonine, at this position occurs naturally in some organisms, further suggesting that sequence variation at this site is not detrimental per se, whereas the introduction of a novel oligosaccharide is (Supplemental Figure 3). Taken together, these data suggest that the A349S mutation leads to a gain of glycosylation, which prevents $\alpha 3 \beta 1$ heterodimerization and cell surface expression.

Heterodimerization and cell surface expression of $\alpha 3^{A 349 S} \beta 1$ are not impaired by association with CD151. Complex formation with CD151 requires an extracellular region in the $\alpha 3$ subunit comprising amino acids 570-705, adjacent to the loop containing alanine 349
(32). Because binding of $\mathrm{CD} 151$ to the $\alpha 3^{\mathrm{A} 349 \mathrm{~S}}$ precursor is not impaired, we hypothesized that the association of CD151 with $\alpha 3^{\mathrm{A} 349 \mathrm{~S}}$ may affect the orientation of the oligosaccharide in such a way that it induces a conformational change, thereby preventing its heterodimerization with $\beta 1$. To explore this possibility, we introduced either $\alpha 3^{\mathrm{WT}}$ or $\alpha 3^{\mathrm{A} 349 \mathrm{~S}}$, but not CD151, in $\alpha 3 /$ CD151-deficient podocytes and investigated their expression at the cell surface by flow cytometry. Similarly to that in podocytes that express CD151, $\alpha 3^{\text {WT }}$ but not $\alpha 3^{\mathrm{A} 349 \mathrm{~S}}$ was expressed at the cell surface in $\mathrm{CD} 151^{-/-}$cells (Figure 7A). Accordingly, in lysates of $C D 151^{-/}$cells reconstituted with $\alpha 3^{\mathrm{WT}}$, both the precursor and mature $\alpha 3$ subunits were detected by Western blotting, while in lysates of $C D 151^{-/-}$cells expressing the mutant $\alpha 3^{\mathrm{A} 349 S}$, only the precursor $\alpha 3$ subunit was present (Figure 7B). These results were further confirmed by the lack of expression of mature $\alpha 3^{\mathrm{A} 349 \mathrm{~S}}$, but not of $\alpha 3^{\mathrm{WT}}$, in podocytes that contain CD151, in which the a3-binding glutamine-arginine-aspartic acid (QRD) at amino acid position 194-196 was mutated to isoleucine-asparagine-phenylalanine (INF) (Figure 7B). This demonstrates that the association of CD151 with $\alpha 3^{\mathrm{A} 349 \mathrm{~S}}$ is not the cause of defective $\alpha 3 \beta 1$ 
A

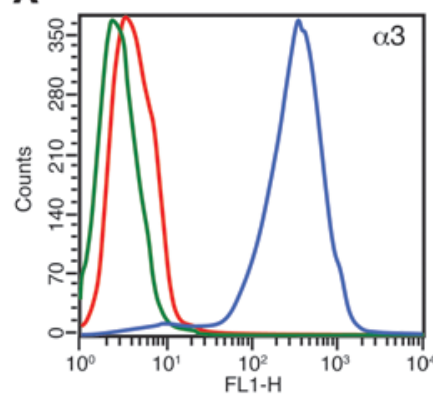

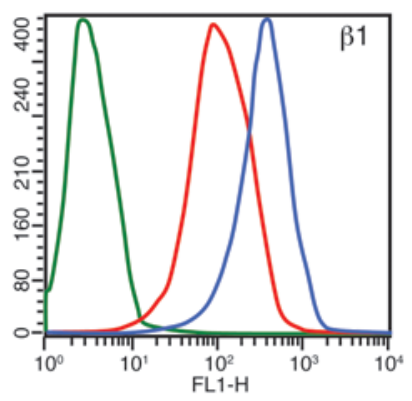

B

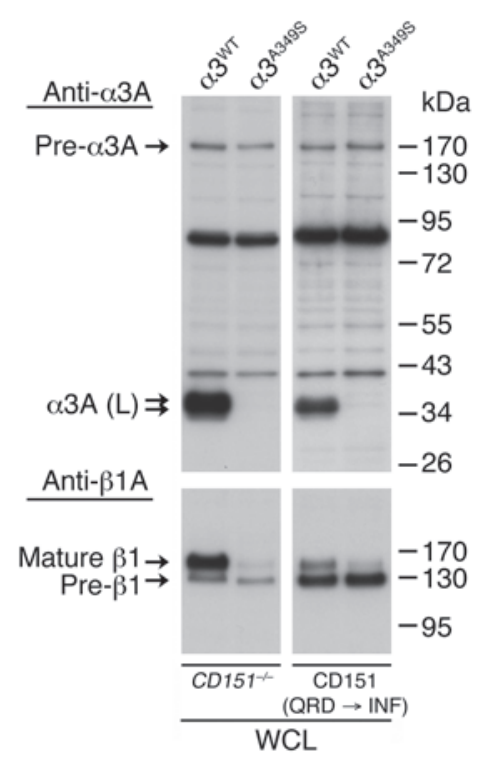

heterodimerization, cleavage of precursor $\alpha 3$, and cell surface expression and suggests that the introduction of an additional oligosaccharide side chain is by itself sufficient to disturb proper folding of the $\alpha 3$ subunit.

The A349S mutation causes accumulation of the $\alpha 3$ and $\beta 1$ precursors in the $E R, \alpha 3$ ubiquitination, and proteasomal degradation. We next investigated the subcellular distribution of $\alpha 3^{\mathrm{WT}} \beta 1$ and $\alpha 3^{\mathrm{A3} 495} \beta 1$ in podocytes by confocal microscopy. First, we used the J143 antibodies against the extracellular domain of $\alpha 3$. As expected, $\alpha 3^{\mathrm{WT}}$ was clearly distributed over the plasma membrane and was enriched laterally at cell-cell contact sites and basally in FAs, as indicated by colocalization with the FA marker phospho-paxillin. In contrast, no $\alpha 3^{\mathrm{A} 349 \mathrm{~S}}$ was detected at the plasma membrane, consistent with the biochemical and flow cytometry data. Furthermore, J143 did not react with precursor $\alpha 3^{\mathrm{A} 349 \mathrm{~S}}$ in intracellular compartments, supporting the idea that this antibody recognizes the $\alpha 3$ subunit only when it is associated with $\beta 1$ (Figure 8A). In the presence of $\alpha 3^{\mathrm{WT}}, \beta 1$ was distributed over the plasma membrane, laterally in cell-cell contacts and basally in FAs, as determined using an antibody directed against phosphorylated paxillin. However, in cells expressing $\alpha 3^{\mathrm{A} 349 S}$, there was hardly any $\beta 1$ detectable at the plasma membrane, although the FA pool was retained. Instead, a dramatic accumulation was observed in a perinuclear compartment, which was barely visible in cells expressing $\alpha 3^{\mathrm{WT}}$ (Figure 8B). These observations are consistent with the dramatic reduction in $\beta 1$ surface expression observed by flow cytometry and the large pool of $\beta 1$ precursor found in lysates of $\alpha 3^{\mathrm{A} 349}$. The perinuclear

\section{Figure 7}

CD151 binding does not impair $\alpha 3 \beta 1$ heterodimerization or transport of $\alpha 3^{\text {A349S }}$ to the plasma membrane. (A) $\alpha 3 / C D 151$-deficient podocytes were reconstituted with $\alpha 3^{\mathrm{WT}}$ (blue) or $\alpha 3^{\mathrm{A} 349 \mathrm{~S}}$ (red), and cell surface expression of $\alpha 3$ and $\beta 1$ was analyzed by flow cytometry. Green indicates $\alpha 3^{W T}$ incubated only with secondary antibody. (B) CD151-deficient podocytes expressing either $\alpha 3^{\mathrm{WT}}$ or $\alpha 3^{\mathrm{A} 349 \mathrm{~S}}$ were lysed, and precursor and mature $\alpha 3$ were detected by Western blotting. Alternatively, $\alpha 3 / C D 151-$ deficient podocytes were reconstituted with $\alpha 3^{W T}$ or $\alpha 3^{A 349 S}$ as well as with CD151, in which the QRD motif was mutated to INF. Podocytes were lysed, and precursor and mature $\alpha 3$ were detected by Western blotting.

distribution points, most likely, to the ER-localized excess of $\beta 1$ that cannot be heterodimerized with $\alpha 3^{\mathrm{A349S}}$. We then analyzed $\alpha 3$ localization using our antibody directed against the cytoplasmic domain of $\alpha 3$. Much like the staining pattern obtained with J143, this antibody detected $\alpha 3^{\mathrm{WT}}$ at the plasma membrane, in cell-cell contacts, and in cell-matrix adhesions. Intriguingly, it also revealed accumulation of $\alpha 3^{\mathrm{A3} 49 \mathrm{~S}}$ around the nucleus, where it is colocalized with $\beta 1$ (Figure 8, C and D). Finally, we examined the distribution of CD151. In cells expressing $\alpha 3^{\mathrm{WT}}$, CD151 is colocalized with $\alpha 3$ at the plasma membrane and in adhesions. In cells expressing $\alpha 3^{\mathrm{A} 349 \mathrm{~S}}$, CD151 was also localized at the plasma membrane, consistent with observations that CD151 can traffic to the cell surface independently of $\alpha 3 \beta 1$ (34). However, a small perinuclear pool colocalized with $\alpha 3^{\mathrm{A} 349 \mathrm{~S}}$, which probably represents CD151 bound to precursor $\alpha 3$ in the ER (Figure 8E). Costaining of $\alpha 3$ or $\beta 1$ with the ER markers protein disulphide isomerase or calnexin confirmed that the perinuclear compartment to which $\alpha 3^{\mathrm{A} 349 \mathrm{~S}} \beta 1$ is localized is indeed the ER (Figure 8F).

Calnexin is a chaperone protein that is involved in ER retention and folding of nascent glycoproteins (35). Both the integrin $\alpha$ and $\beta$ subunit precursors undergo several cycles of folding and unfolding, during which calnexin associates with and dissociates from both to ensure that they reach their proper conformation before being transported to the Golgi (36). To investigate whether $\alpha 3^{\mathrm{A} 349 \mathrm{~S}}$ could still associate with calnexin, we precipitated either $\beta 1$ or $\alpha 3$ from podocytes expressing $\alpha 3^{\mathrm{WT}}$ or $\alpha 3^{\mathrm{A} 349 \mathrm{~S}}$. Both wildtype and mutant $\alpha 3$, as well as $\beta 1$, coprecipitated from podocyte lysates with calnexin, indicating that the interaction of calnexin was not impaired with either subunit (Supplemental Figure 4). In an attempt to circumvent the quality control mechanism in the $\mathrm{ER}$, we cultured podocytes at $30^{\circ} \mathrm{C}$ or incubated them with the pharmacological chaperones curcumin or 4-phenylbutyrate. Compounds that can act as chaperones have been used to evade the ER response to misfolded $G$ protein-coupled receptors, including the vasopressin receptor, gonadotropin-releasing hormone receptor, and cystic fibrosis transmembrane conductance regulator $(37,38)$. Therefore, pharmacological chaperones are suggested as treatment for a variety of diseases due to protein misfolding (39). However, neither strategy induced cell surface expression of $\alpha 3^{\mathrm{A349S}}$, whereas the cell surface expression of $\alpha 3^{\mathrm{WT}}$ was increased (C. Margadant, unpublished observations).

Misfolded proteins are disposed of by the ER-associated degradation pathway, which involves ubiquitination and transport from the ER to the cytosol for proteasomal degradation $(40,41)$. To inhibit the proteasome, podocytes were incubated for 6 hours with lactacystin $(10 \mu \mathrm{M})$. Treatment with lactacystin induced a striking accumulation of polyubiquitinated $\alpha 3^{\mathrm{A} 349 \mathrm{~S}}$ precursor, but much less 
A

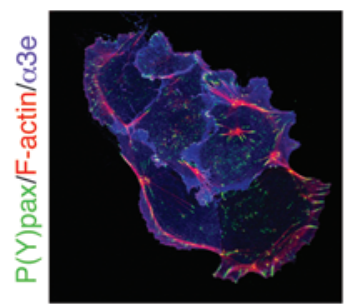

B

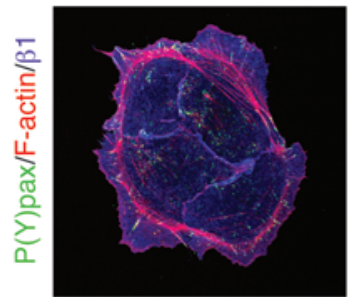

C

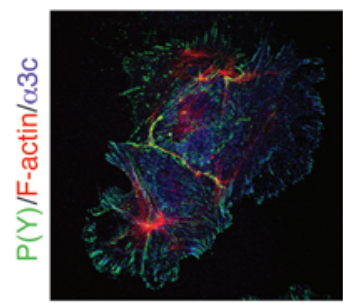

D

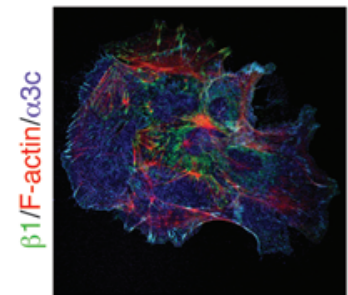

E

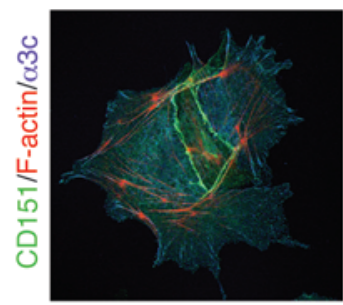

$\alpha 3^{W T}$
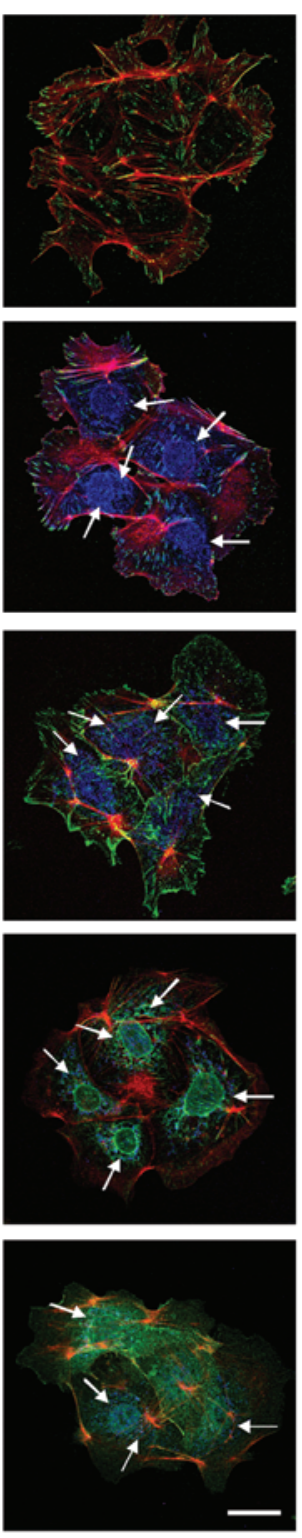

$\alpha 3^{\text {A349S }}$
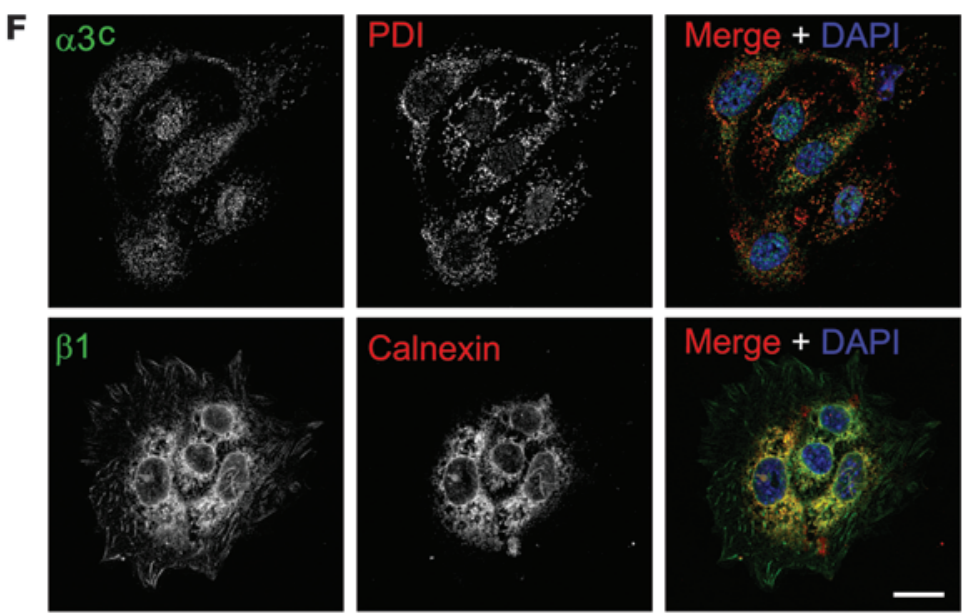

G
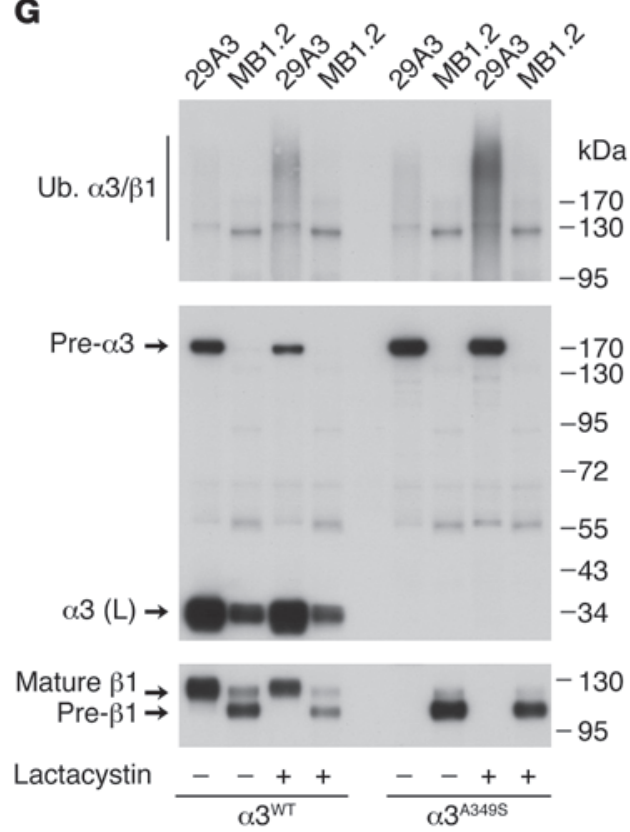

Figure 8

The $\alpha 3^{\text {A349S }}$ mutation causes accumulation of $\beta 1$ precursor in the ER and ubiquitination and proteasomal degradation of $\alpha 3$. (A) Subcellular localization of $\alpha 3$ was investigated in podocytes expressing either $\alpha 3^{\mathrm{WT}}$ or $\alpha 3^{\mathrm{A} 349 \mathrm{~S}}$, using J143 against the extracellular domain of $\alpha 3$ (blue). Filamentous actin (F-actin) was stained with phalloidin (red), and FAs were visualized using an antibody against phosphorylated paxillin $[P(Y)$ pax; green]. (B) Localization of $\beta 1$ (blue) in podocytes expressing either $\alpha 3^{W T}$ or $\alpha 3^{A 349 S}$. Green indicates phosphorylated paxillin, and red indicates F-actin. (C) Distribution of $\alpha 3$ was investigated using an antibody directed against the cytoplasmic domain (blue), and FAs were detected with an antibody against phosphotyrosines [P(Y); green]. Red indicates F-actin. (D) Colocalization of $\beta 1$ (green) and $\alpha 3$ (blue). Red indicates F-actin. (E) Distribution of CD151 (green) and $\alpha 3$ (blue). Red indicates F-actin. (A-E) Scale bar: $20 \mu \mathrm{m}$. Arrows indicate perinuclear localization of $\alpha 3^{A 349 S}$ and $\beta 1$ in each panel. (F) Colocalization of $\alpha 3^{A 349 S}$ with protein disulphide isomerase (PDI; top) and $\beta 1$ with calnexin (bottom). Nuclei were stained with DAPI. Scale bar: $20 \mu \mathrm{m}$. (G) Podocytes expressing either $\alpha 3^{\mathrm{WT}}$ or $\alpha 3^{\mathrm{A} 349 \mathrm{~S}}$ were treated for 6 hours with the proteasomal inhibitor lactacystin $(10 \mu \mathrm{M})$ and lysed, and ubiquitinated $\alpha 3 \beta 1$ was detected by precipitation of the indicated subunits, followed by Western blotting.

of $\alpha 3^{\mathrm{WT}}$, indicating that a large fraction of the mutant precursor is indeed ubiquitinated and proteasomally degraded (Figure 8G).

Together, these data demonstrate that the misfolded $\alpha 3^{\mathrm{A} 349 \mathrm{~S}}$ precursor is cleared by ubiquitination and subsequent proteasomal degradation, whereas the excess of $\beta 1$ that is unable to associate with $\alpha 3^{\mathrm{A} 349 \mathrm{~S}}$ is mostly retained in the ER.

\section{Discussion}

Here, we report on a patient with a novel missense mutation in the ITGA3 gene, for which she is homozygous and which disturbs the biosynthesis of integrin $\alpha 3 \beta 1$, resulting in a multiorgan disorder, consisting of interstitial lung disease and congenital nephrotic syndrome. The mutation causes a gain of glycosylation in the $\alpha 3$ 


\section{A}

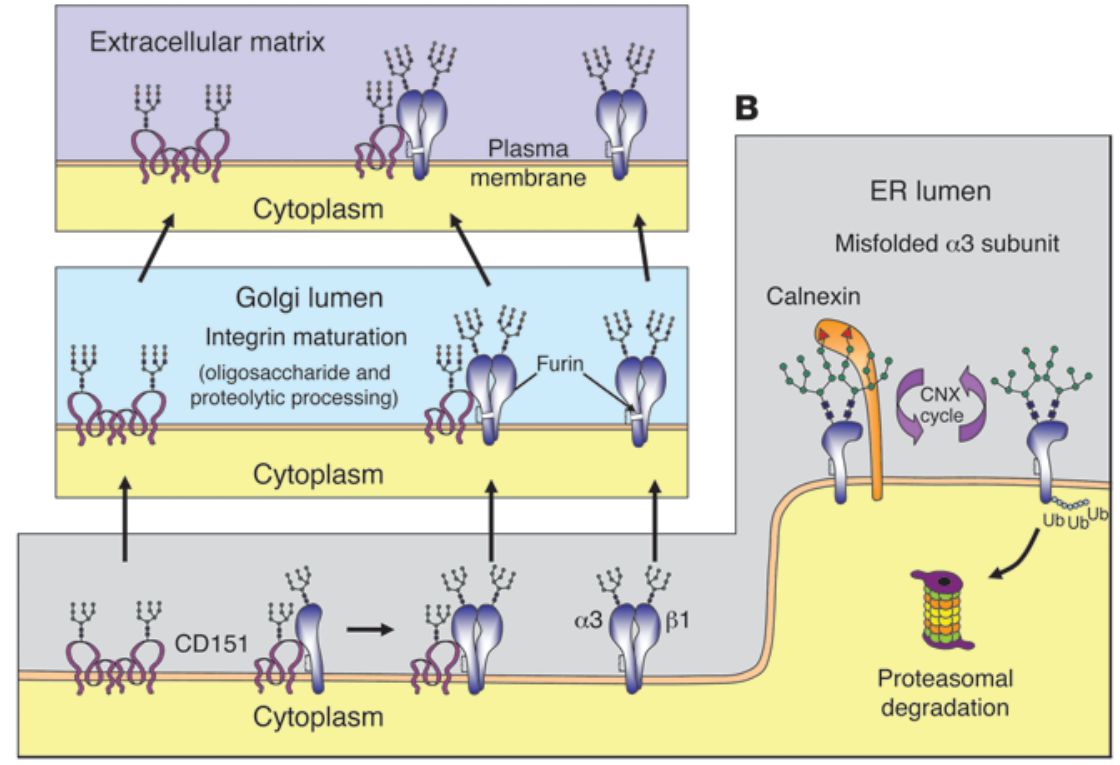

\section{Figure 9}

Model summarizing the biosynthetic route of CD151 and wild-type and mutant integrin $\alpha 3 \beta 1$. Newly synthesized $\alpha 3$ and $\beta 1$ precursors, as well as CD151, undergo $N$-glycosylation and folding in the ER, facilitated by the chaperone calnexin. Thereafter, CD151 can associate with other tetraspanins or associate with the $\alpha 3$ subunit prior to $\alpha / \beta$ heterodimerization. Properly folded and assembled complexes then traffic to the Golgi apparatus as integrin $\alpha 3 \beta 1$ heterodimers, $\alpha 3 \beta 1$ heterodimers in association with CD151, or CD151 in complex with other tetraspanins. (A) In the Golgi, the high-mannose oligosaccharides are processed to complex type sugars, and the $\alpha 3$ subunit is cleaved into a heavy and a light chain to generate the mature $\alpha 3 \beta 1$ heterodimer. (B) Gain-of-glycosylation mutations in an integrin subunit can cause a failure to reach the final conformation and thereby prevent $\alpha / \beta$ heterodimerization. The affected subunits are then cleared by the ubiquitin/proteasome system, whereas their partners accumulate in the ER. subunit, which prevents heterodimerization with $\beta 1$ but not the association with CD151. Although the mutant $\alpha 3$ subunit still interacts with calnexin, its misfolding is terminal, leading to rapid clearance by the ubiquitin/proteasome system while the noncomplexed $\beta 1$ accumulates in the ER (Figure 9).

The kidney and lung abnormalities observed in the patient are consistent with the defects in kidney and lung morphogenesis observed in $\alpha 3$-deficient mice (13). In addition, we found extracapillary proliferation, focal segmental glomerulosclerosis, and interstitial fibrosis in the glomeruli of the patient, which was not observed in $\alpha 3$-null mice. The latter observations likely reflect the progressive nature of the glomerular damage, as $\alpha 3$-deficient mice die much earlier after birth. In addition to the kidney and lung defects, absence of $\alpha 3$ in the epidermis causes skin abnormalities, including (micro-)blistering, basement membrane duplication, inflammation, and alopecia (13-17). Although obvious macroscopic skin abnormalities were not observed in the patient described here, we cannot exclude that microblistering or aberrant epidermal basement membrane organization occurred.

During the revision of this article, a report was published describing mutations in ITGA3. While the kidney and lung defects observed in the patients concerned are largely similar to those described here, an abnormal skin phenotype was also reported (42). It should be noted that the skin defects caused by $\alpha 3$ deficiency in mice are relatively minor, because adhesion of epidermal keratinocytes to LNs is rescued by other integrins, most notably the hemidesmosome-based integrin $\alpha 6 \beta 4(17,43)$. Thus, redundant mechanisms exist in epidermal keratinocytes to ensure adhesion, which is probably why skin defects are not always detected in the absence of $\alpha 3$ (44). Similarly, a mutation in $C D 151$ has been described to cause epidermolysis bullosa, whereas skin defects have not been observed in Cd151-deficient mice $(19,24,25,28)$. Nevertheless, $\alpha 6$ integrins cannot compensate for the absence of $\alpha 3 \beta 1$ in the kidneys and the lungs, even though $\alpha 6$ expression seemed to be increased in these organs in the patient described here.
Our immunoprecipitation experiments yield some important conclusions. First, the mutant $\alpha 3$ subunit does not heterodimerize with $\beta 1$, as shown by pull-down of either the $\alpha 3$ subunit with 29A3 or the $\beta 1$ subunit with MB1.2. Intriguingly, the 9EG7 antibody, which is widely used as a marker for $\beta 1$ integrins in the active, high-affinity conformation (45), exclusively precipitated precursor $\beta 1$ but no mature $\beta 1$. In addition, 9EG7 did not recognize $\beta 1$ on the cell surface by flow cytometry (C. Margadant, unpublished observations). These observations suggest that there is no $\beta 1$ in its active conformation at the cell surface, questioning the use of 9EG7 as a bona fide marker for active $\beta 1$ integrins in general. Second, the J143 and P1B5 antibodies, both directed against the extracellular domain of $\alpha 3$, apparently recognize a complex-dependent epitope on the $\alpha 3$ subunit, because they do not precipitate precursor $\alpha 3^{\mathrm{A} 349 \mathrm{~S}}$, and precipitate much smaller amounts of the $\alpha 3^{\mathrm{WT}}$ precursor than 29A3, but comparable amounts of mature $\alpha 3$ or $\beta 1$. The fraction of precursor $\alpha 3$ that is not recognized by these antibodies is presumably not associated with $\beta 1$. Third, our data show that CD151 can associate with both the $\alpha 3$ precursor and mature $\alpha 3$, which is consistent with previous observations, indicating that CD151- $\alpha 3$ association occurs early during biosynthesis of $\alpha 3 \beta 1$ (34). In addition, it has been shown that CD151 mutants that are not expressed at the cell surface can be coprecipitated with $\alpha 3$, suggesting that CD151 binds intracellular $\alpha 3$, of which at least a fraction consists of precursor (34). In fact, the observation that CD151 binds precursor $\alpha 3^{\mathrm{A} 349 \mathrm{~S}}$, even though the latter does not associate with $\beta 1$, suggests that CD151- $\alpha 3$ association occurs prior to $\alpha / \beta$ heterodimerization.

The A349S mutation leads to hyperglycosylation of the $\alpha 3$ precursor, which is likely the cause of its disruptive effects, as (a) considerable sequence variability exists at this position among eukaryotes, but a serine or threonine is never encountered, and (b) a mutant carrying a glycine at this position, which cannot be glycosylated, associates normally with $\beta 1$ and is expressed at the cell surface. As the novel sugar moiety is oriented away from the $\alpha / \beta$ interface, a direct effect on $\alpha / \beta$ subunit interaction by steric hindrance seems unlikely. However, it is located between 
2 FG-GAP repeats, which form $\beta$-strands in the $\beta$-propeller domain. This region is very important for the proper folding of integrin $\alpha$ subunits, and numerous mutations in the $\beta$-propeller of $\alpha$ IIb have been identified that disturb its folding and the heterodimerization with $\beta 3$ (46). Similar to what we have observed, such mutations lead to proteasomal degradation of the mutant $\alpha I I b$ precursor, whereas the noncomplexed $\beta 3$ precursor is retained in the ER (47).

The repertoire of gain-of-glycosylation disorders is everexpanding, and gain-of-glycosylation mutations that disrupt integrin biosynthesis as the cause for a human disorder have previously been identified in the genes encoding integrin subunits $\beta 2$ (causing leukocyte adhesion deficiency) and $\beta 3$ (causing Glanzmann's thrombasthenia) (48-50). We now report a new gain-of-glycosylation mutation that prevents the biosynthesis of integrin $\alpha 3 \beta 1$, causing interstitial lung disease and congenital nephrotic syndrome. It will be important to implement ITGA3 gene sequencing in DNA diagnostics for newborns presenting with severe respiratory distress and/or congenital nephrotic syndrome of unknown etiology to facilitate early diagnosis in patients and provide recurrence risk estimation for patients and their relatives.

\section{Methods}

Materials. The cDNA encoding CD151 was provided by M. Hemler (Dana-Farber Cancer Institute, Boston, Massachusetts, USA), and the cDNA encoding human $\alpha 3$ has been described previously (51). Mouse mAbs directed against human $\alpha 3$ were J143 (hybridoma from ATCC) and P1B5 (W. Carter, Fred Hutchinson Cancer Research Center, Seattle, Washington, USA). Mouse antibodies against human CD151 were 5C11 (F. Berditchevski, University of Birmingham, Birmingham, United Kingdom), TS151R (E. Rubinstein, Hôpital Paul Brousse, Villejuif, France), and 8C3 (K. Sekiguchi, Osaka University, Osaka, Japan). The mouse mAb 29A3 against the cytoplasmic domain of $\alpha 3 \mathrm{~A}$ has been described previously (52). Rat mAbs were GoH3 against $\alpha 6$ and MB1.2 against $\beta 1$ (B. Chan, University of Ontario, Ontario, Canada). Rabbit polyclonal antibodies were directed against the cytoplasmic domain of $\alpha 3 \mathrm{~A}$ (19), the extracellular domain of $\alpha 6$ (AA6NT, ref. 53) (A. Cress, University of Arizona, Tucson, Arizona, USA), the cytoplasmic domains of $\beta 1 A$ and $\alpha 2$ (G. Tarone, University of Turin, Turin, Italy), calnexin (I. Braakman, University of Utrecht, Utrecht, The Netherlands), collagen IV (E. Engvall, The Burnham Institute, La Jolla, California, USA), LN and nidogen (T. Sasaki, Shriners Hospital for Children Research Center, Portland, Oregon, USA), and podocin (C. Antignac, Cochin Biomedical Research Institute, Paris, France). The goat antibody used against mouse $\alpha 3$ was AF2787 obtained from R\&D Systems. TRITCand FITC-conjugated secondary antibodies, phalloidin, and DAPI were obtained from Molecular Probes. HRP-conjugated secondary antibodies were obtained from Amersham; curcumin, 4-phenylbutyrate, tunicamycin, and zeocin were obtained from Sigma-Aldrich; and collagen I was obtained from Vitrogen (Nutacon).

Patient material and DNA diagnostics. Peripheral blood samples were obtained from the patient and her parents and sibling after informed consent was given. Genomic DNA was extracted from these samples, and known genes implicated in nephrotic syndrome (NPHS1, WT1, PLCE1, LAMB2, NPHS2, TRPC6, ACTN4, INF2, ABCA3, TTF1, TTF2) and neonatal respiratory distress $(A B C A 3, S F T P B$, and $S F T P C)$ were screened for variants by Sanger sequencing. Tissue samples used in this study were derived from kidney and lung autopsy or biopsy specimens, obtained with informed parental consent.
SNP array analysis. Genomic DNA was obtained from peripheral blood samples of the patient and her parents. CNV screening by means of microarray analyses was carried out on the Affymetrix GeneChip 250k (NspI) SNP array platform (Affymetrix Inc.), which contains 25-mer oligonucleotides representing a total of 262,264 SNPs. The average resolution of this array platform is $150-200 \mathrm{~kb}$. Hybridizations were performed according to the manufacturer's protocols. CNVs and long continuous stretch of homozygosity were determined using Copy Number Analyzer for Affymetrix GeneChip mapping software (CNAG version 2.0). The average resolution of this array platform is $150-200 \mathrm{~Kb}$. To investigate uniparental disomy, genotypes were called by Affymetrix Genotype Console Software v2.1.

Mutation analysis of ITGA3. To sequence the complete coding region of ITGA3 in genomic DNA, exon-flanking primers were designed with the Primer3 program (Supplemental Table 1), which is available online (http:// frodo.wi.mit.edu/). All coding exons of ITGA3 were amplified by PCR in a total reaction volume of $20 \mu \mathrm{l}$, which contained $50 \mathrm{ng}$ DNA, $10 \mathrm{pmol}$ of each primer, $50 \mathrm{nmol}$ of $\mathrm{MgCl}_{2}$, 1x Taq buffer (Applied Biosystems Inc.), and $1 \mathrm{U}$ Taq polymerase (Applied Biosystems Inc.). PCR products were sequenced in forward and reverse directions by Sanger sequencing on a 3730 DNA analyzer (Applied Biosystems Inc.) with dye-termination chemistry (Big Dye Terminator Cycle Sequencing Kit, version 1.1; Applied Biosystems Inc.). Sequence analysis was performed using Sequencher 4.8 software. The identified gene variant was confirmed by bidirectional sequencing. Segregation analysis was performed by Sanger sequencing. Furthermore, we sequenced the ITGA3 gene in 192 healthy blood donors from The Netherlands. To determine whether the mutation was previously detected in reference populations, we used our in-house database of approximately 100 whole-exome sequencing experiments, dbSNP, and the Exome Variant Server of the NHLBI Exome Sequencing Project, which is available online (http://evs.gs.washington.edu/EVS/).

Generation of cell lines, cell culture, cloning, and retroviral transduction. Glomeruli were isolated from $\operatorname{Itg} a 3^{f / / f l} \mathrm{Cd} 151^{f / / f l} \operatorname{Tr} p 53^{+/-}$mice, according to the Dynabead perfusion method as described previously (29), and cultured on $3 \mu \mathrm{g} / \mathrm{ml}$ collagen I at $37^{\circ} \mathrm{C}$ and $5 \% \mathrm{CO}_{2}$ in keratinocyte serum-free medium (K-SFM; Gibco BRL) supplemented with $50 \mu \mathrm{g} / \mathrm{ml}$ bovine pituitary extract, $5 \mathrm{ng} / \mathrm{ml} \mathrm{EGF}, 100 \mathrm{U} / \mathrm{ml}$ penicillin, and $100 \mathrm{U} / \mathrm{ml}$ streptomycin. Outgrowing epithelial cells were sorted for expression of podocalyxin and absence of CD31. The Itga 3 and $C d 151$ genes were deleted by adenoviral delivery of Cre-recombinase. The A349S and A349G mutations were generated by PCR overlap extension method, using a cDNA encoding human full-length $\alpha 3 \mathrm{~A}$ as a template. Wild-type ITGA3 was isolated by digestion with SacI and ligated into pUC18-a3. After digestion with SphI, ITGA3 was ligated into LZRS-IRES-zeo, which was transfected into Phoenix packaging cells using the calcium phosphate method. Virus-containing supernatant was isolated after 48 hours, and stable expression in the $\alpha 3 / C D 151$-deficient podocytes was achieved by retroviral transduction, followed by selection with zeocin $(200 \mu \mathrm{g} / \mathrm{ml})$. The podocytes were also reconstituted with a cDNA encoding FLAG-tagged human CD151.

Immunoprecipitation and Western blotting. Cell lysis and immunoprecipitation were performed essentially as described previously (17). Whole cell lysates and precipitates were resolved by SDS-PAGE, transferred to polyvinylidene difluoride membranes (Millipore), and analyzed by Western blotting. Bound antibodies were detected using the ECL detection system from GE Healthcare.

Immunobistochemistry, electron microscopy, confocal microscopy, and flow cytometry. Electron microscopy and immunohistochemistry were performed using standard procedures. For indirect immunofluorescence, cryosections from patient biopsies or coverslips with cells were incubated with antibodies as previously described (17). Images were acquired at room 
temperature with a confocal Leica TCS NT or AOBS microscope using $\times 20$ dry (numerical aperture 0.7$), \times 40$ oil (numerical aperture 1.25 ), and $\times 63$ oil (numerical aperture 1.32) objectives (Leica) and AxioVision 4 software (Carl Zeiss MicroImaging). Pictures were processed using Photoshop 7.0 and ImageJ. Flow cytometry and cell sorting were performed as previously described (17).

Statistics. The 250k Affymetrix microarray data was analyzed using CNAG version 2.0 as previously described (54). CNVs were detected on the normalized $\log _{2}$ ratios using a 5 -state hidden Markov model with default settings $(-1 \mu,-0.3 \mu, 0 \mu, 0.38 \mu, 0.55 \mu)$. These settings were deemed appropriate as the autosomal standard deviation and the sex-mismatch mean ChrX were within the quality control ranges (less than 0.2 and greater than 0.3 , respectively). Analysis for loss of heterozygosity ( $\mathrm{LOH}$ ) was also performed using CNAG version 2.0. This algorithm uses a maximum likelihood calculation, with an inference based on the frequencies of heterozygous SNPs to determine significant stretches of $\mathrm{LOH}$. A log-likelihood cut-off value of 15 was used to identify regions of $\mathrm{LOH}$.

Study approval. Written informed consent for SNP array analysis and Sanger sequencing as a part of the diagnostic process (approved by the Medical Ethical Committee of the University Medical Center Utrecht) was obtained from the parents. All studies involving mice were approved by the animal ethics board of The Netherlands Cancer Institute.

\section{Acknowledgments}

We are grateful to C. Antignac, I. Braakman, F. Berditchevski, B. Chan, W. Carter, A. Cress, E. Engvall, M. Hemler, E. Rubenstein, T. Sasaki, K. Sekiguchi, and G. Tarone for their generous gifts of reagents. We thank A. Perrakis for help with the modeling of the $\alpha 3 \beta 1$ ectodomain and A.Y. Konijnenberg, E.J. D'Haens, R.A. van Lingen, and G. Shabo for taking care of the patient. This work was financially supported by the Dutch Kidney Foundation, with a grant to K.Y. Renkema (KSTP10.004) and A. Sonnenberg (C07.2232).

Received for publication March 30, 2012, and accepted in revised form September 6, 2012.

Address correspondence to: Kirsten Y. Renkema, Department of Medical Genetics, STR 1.305, University Medical Center Utrecht, PO Box 85060, 3508 AB Utrecht, The Netherlands. Phone: 31.88.7568312; Fax: 31.88.7553801; E-mail: k.renkema@ umcutrecht.nl. Or to: Arnoud Sonnenberg, Division of Cell Biology, The Netherlands Cancer Institute, Plesmanlaan 121, 1066 CX Amsterdam, The Netherlands. Phone: 31.20.5121942; Fax: 31.20.5121944; E-mail: a.sonnenberg@nki.nl.
1. Campbell ID, Humphries MJ. Integrin structure, activation, and interactions. Cold Spring Harb Perspect Biol. 2011;3(3):pii:a004994.

2. Geiger B, Yamada KM. Molecular architecture and function of matrix adhesions. Cold Spring Harb Perspect Biol. 2011;3(5):pii:a005033.

3. Kim C, Ye F, Ginsberg MH. Regulation of integrin activation. Annu Rev Cell Dev Biol. 2011;27:321-345.

4. Caswell PT, Vadrevu S, Norman JC. Integrins: masters and slaves of endocytic transport. Nat Rev Mol Cell Biol. 2009;10(12):843-853

5. Margadant C, Monsuur HN, Norman JC, Sonnenberg A. Mechanisms of integrin activation and trafficking. Curr Opin Cell Biol. 2011;23(5):607-614.

6. Lissitzky JC, et al. Endoproteolytic processing of integrin pro-alpha subunits involves the redundant function of furin and proprotein convertase (PC) $5 \mathrm{~A}$, but not paired basic amino acid converting enzyme (PACE) 4, PC5B or PC7. Biochem J. 2000; 346:133-138

7. Delwel GO, Kuikman I, van der Schors RC, de Melker AA, Sonnenberg A. Identification of the cleavage sites in the alpha6 A integrin subunit: structural requirements for cleavage and functional analysis of the uncleaved alpha6Abeta1 integrin. Biochem J. 1997;324:263-272.

8. Pulkkinen L, Uitto J. Mutation analysis and molecular genetics of epidermolysis bullosa. Matrix Biol. 1999;18(1):29-42.

9. Nurden AT, Fiore M, Nurden P, Pillois X. Glanzmann thrombasthenia: a review of ITGA2B and ITGB3 defects with emphasis on variants, phenotypic variability, and mouse models. Blood. 2011; 118(23):5996-6005.

10. Hayashi YK, et al. Mutations in the integrin alpha7 gene cause congenital myopathy. Nat Genet. 1998; 19(1):94-97.

11. Hogg N, Patzak I, Willenbrock F. The insider's guide to leukocyte integrin signalling and function. Nat Rev Immunol. 2011;11(6):416-426.

12. Wickström SA, Radovanac K, Fässler R. Genetic analyses of integrin signaling. Cold Spring Harb Perspect Biol. 2011;3(2):pii:a005116.

13. Kreidberg JA, et al. Alpha3beta1 integrin has a crucial role in kidney and lung organogenesis. Development. 1996;122(11):3537-3547.

14. DiPersio CM, Hodivala-Dilke KM, Jaenisch R, Kreidberg JA, Hynes RO. alpha3beta1 integrin is required for normal development of the epidermal basement membrane. J Cell Biol. 1997; 137(3):729-742.

15. Hodivala-Dilke KM, DiPersio CM, Kreidberg JA, Hynes RO. Novel roles for alpha3beta1 integrin as a regulator of cytoskeletal assembly and as a trans-dominant inhibitor of integrin receptor function in mouse keratinocytes. J Cell Biol. 1998; 142(5):1357-1369.

16. Conti FJ, Rudling RJ, Robson A, Hodivala-Dilke KM. alpha3beta1-integrin regulates hair follicle but not interfollicular morphogenesis in adult epidermis. J Cell Sci. 2003;116(pt 13):2737-2747.

17. Margadant C, Raymond K, Kreft M, Sachs N, Janssen $\mathrm{H}$, Sonnenberg A. Integrin alpha3beta1 inhibits directional migration and wound re-epithelialization in the skin. J Cell Sci. 2009;122(pt 2):278-288.

18. Yauch RL, Berditchevski F, Harler MB, Reichner J, Hemler ME. Highly stoichiometric, stable, and specific association of integrin $\alpha 3 \beta 1$ with CD151 provides a major link to phosphatidylinositol 4-kinase, and may regulate cell migration. Mol Biol Cell. 1998;9(10):2751-2765.

19. Sachs N, et al. Kidney failure in mice lacking the tetraspanin CD151. J Cell Biol. 2006;175(1):33-39.

20. El-Aouni C, et al. Podocyte-specific deletion of integrin-linked kinase results in severe glomerular basement membrane alterations and progressive glomerulosclerosis. J Am Soc Nephrol. 2006; 17(5):1334-1344

21. Miner JH, Li C. Defective glomerulogenesis in the absence of laminin alpha5 demonstrates a developmental role for the kidney glomerular basement membrane. Dev Biol. 2000;217(2):278-289.

22. Kanasaki K, et al. Integrin beta1-mediated matrix assembly and signaling are critical for the normal development and function of the kidney glomerulus. Dev Biol. 2008;313(2):584-593.

23. Pozzi A, et al. Beta1-integrin expression by podocytes is required to maintain glomerular structural integrity. Dev Biol. 2008;316(2):288-301.

24 . Sachs N, et al. Blood pressure influences end-stage renal disease of Cd151 knockout mice. J Clin Invest. 2012;122(1):348-358

25. Baleato RM, Guthrie PL, Gubler MC, Ashman LK, Roselli S. Deletion of CD151 results in a strain-dependent glomerular disease due to severe alterations of the glomerular basement membrane. Am
J Pathol. 2008;173(4):927-937.

26. Kaplan JM, et al. Mutations in ACTN4, encoding alpha-actinin-4, cause familial focal segmental glomerulosclerosis. Nat Genet. 2000;24(3):251-256.

27. Zenker M, et al. Human laminin beta2 deficiency causes congenital nephrosis with mesangial sclerosis and distinct eye abnormalities. Hum Mol Genet. 2004;13(21):2625-2632.

28. Karamatic Crew V, et al. CD151, the first member of the tetraspanin (TM4) superfamily detected on erythrocytes, is essential for the correct assembly of human basement membranes in kidney and skin. Blood. 2004;104(8):2217-2223.

29. Takemoto M, et al. A new method for large scale isolation of kidney glomeruli from mice. Am J Pathol. 2002;161(3):799-805.

30. Kazarov AR, Yang X, Stipp CS, Sehgal B, Hemler ME. An extracellular site on tetraspanin CD151 determines alpha3 and alpha6 integrin-dependent cellular morphology. J Cell Biol. 2002;158(7):1299-1309.

31. Serru V, et al. Selective tetraspan-integrin complexes (CD81/alpha4beta1, CD151/alpha3beta1, CD151/ alpha6beta1) under conditions disrupting tetraspan interactions. Biochem J. 1999;340(pt 1):103-111.

32. Yauch RL, Kazarov AR, Desai B, Lee RT, Hemler ME. Direct extracellular contact between integrin alpha(3)beta(1) and TM4SF protein CD151. J Biol Chem. 2000;275(13):9230-9238.

33. Nagae M, et al. Crystal structure of $\alpha 5 \beta 1$ integrin ectodomain: Atomic details of the fibronectin receptor. J Cell Biol. 2012;197(1):131-140.

34. Berditchevski F, Gilbert E, Griffiths MR, Fitter S, Ashman L, Jenner SJ. Analysis of the CD151-alpha3beta 1 integrin and CD151-tetraspanin interactions by mutagenesis. J Biol Chem. 2001; 276(44):41165-41174

35. Caramelo JJ, Parodi AJ. Getting in and out from calnexin/calreticulin cycles. J Biol Chem. 2008; 283(16):10221-10225

36. Lenter M, Vestweber D. The integrin chains beta1 and alpha 6 associate with the chaperone calnexin prior to integrin assembly. J Biol Chem. 1994; 269(16):12263-12268

37. Egan ME, et al. Curcumin, a major constituent of turmeric, corrects cystic fibrosis defects. Science. 2004;304(5670):600-602.

38. Wang X, Koulov AV, Kellner WA, Riordan JR, Balch WE. Chemical and biological folding contribute to 
temperature-sensitive DeltaF508 CFTR trafficking. Traffic. 2008;9(11):1878-1893.

39. Bernier V, Bichet DG, Bouvier M. Pharmacological chaperone action on G-protein-coupled receptors. Curr Opin Pharmacol. 2004;4(5):528-533.

40. Smith MH, Ploegh HL, Weissman JS. Road to ruin: targeting proteins for degradation in the endoplasmic reticulum. Science. 2011;334(6059):1086-1090.

41. Lederkremer GZ. Glycoprotein folding, quality control and ER-associated degradation. Curr Opin Struct Biol. 2009;19(5):515-523.

42. Has $C$, et al. Integrin $\alpha 3$ mutations with kidney, lung, and skin disease. $N$ Engl J Med. 2012; 366(16):1508-1514.

43. Margadant C, Frijns E, Wilhelmsen K, Sonnenberg A. Regulation of hemidesmosome (dis-)assembly by growth factor receptors. Curr Opin Cell Biol. 2008; 20(5):589-596.

44. Margadant C, Charafeddine RA, Sonnenberg A. Unique and redundant functions of integrins in the epidermis. FASEB J. 2010;24(11):4133-4152.
45. Byron A, Humphries JD, Askari JA, Craig SE, Mould AP, Humphries MJ. Anti-integrin monoclonal antibodies. J Cell Sci. 2009;122(pt 22):4009-4011.

46. Nelson EJ, Li J, Mitchell WB, Chandy M, Srivastava A, Coller BS. Three novel beta-propeller mutations causing Glanzmann thrombasthenia result in production of normally stable pro-alphaIIb, but variably impaired progression of pro-alphaIIbbeta 3 from endoplasmic reticulum to Golgi. J Thromb Haemost. 2005;3(12):2773-2783.

47. Mitchell WB, Li J, French DL, Coller BS. alphaIIbbeta3 biogenesis is controlled by engagement of alphaIIb in the calnexin cycle via the N15-linked glycan. Blood. 2006;107(7):2713-2719.

48. Vogt G, Vogt B, Chuzhanova N, Julenius K, Cooper DN, Casanova JL. Gain-of-glycosylation mutations. Curr Opin Genet Dev. 2007;17(3):245-251.

49 . Vogt $\mathrm{G}$, et al. Gains of glycosylation comprise an unexpectedly large group of pathogenic mutations. Nat Genet. 2005;37(7):692-700.

50. Back AL, Kerkering M, Baker D, Bauer TR, Embree
LJ, Hickstein DD. A point mutation associated with leukocyte adhesion deficiency type 1 of moderate severity. Biochem Biophys Res Commun. 1993; 193(3):912-918

51. Delwel GO, et al. Distinct and overlapping ligand specificities of the alpha3Abeta1 and alpha6Abeta 1 integrins: recognition of laminin isoforms. Mol Biol Cell. 1994;5(2):203-215.

52. De Melker AA, et al. The A and B variants of the alpha3 integrin subunit: tissue distribution and functional characterization. Lab Invest. 1997; 76(4):547-563.

53. Ports MO, Nagle RB, Pond GD, Cress AE. Extracellular engagement of alpha6 integrin inhibited urokinase-type plasminogen activator-mediated cleavage and delayed human prostate bone metastasis. Cancer Res. 2009;69(12):5007-5014

54. Nannya $Y$, et al. A robust algorithm for copy number detection using high-density oligonueclotide single nucleotide polymorphism genotyping arrays. Cancer Res. 2005;65(14):6071-6079. 